\title{
An exact sequence for Legendrian links
}

\author{
ANAHITA ESLAMI RAD
}

\begin{abstract}
We obtain an exact sequence of cyclic Legendrian homology for Legendrian links. We present some examples in 3 dimensions and higher. In higher dimensions we count holomorphic curves via Morse flow trees developed by Ekholm.
\end{abstract}

53D42; 57R17

\section{Introduction}

A result of Bourgeois, Ekholm and Eliashberg [4] describes the linearized contact homology of the boundary of a symplectic cobordism obtained by Legendrian surgery in terms of the cyclic homology of the composable part of the Legendrian homology algebra of the Legendrian attaching spheres. In this work we consider the case when the attaching locus has multiple components, describe the relevant version of the homology algebra of the Legendrian attaching spheres remaining after surgery along one sphere, and we obtain an exact sequence for the original link.

Let us recall some basic concepts from contact topology. Let $Y$ be a $(2 n-1)-$ dimensional contact manifold equipped with a maximally nonintegrable field of tangent hyperplanes $\xi$. A Legendrian submanifold $\Lambda \subset Y$ is an $(n-1)$-submanifold of $Y$ which is tangent to $\xi$ everywhere.

Weinstein's description of contact surgery on $Y$ along a Legendrian sphere $\Lambda \subset Y$ gives a new contact manifold $Y_{\Lambda}$ and a symplectic cobordism $X$ from $Y$ to $Y_{\Lambda}$; see Weinstein [20]. In this construction one performs surgery along the Legendrian sphere $\Lambda$ with trivial conformal symplectic normal bundle in the contact manifold $Y$. The resulting manifold $Y_{\Lambda}$ carries again a contact structure which coincides with the old one outside the neighborhood where surgery takes place. The symplectic cobordism between the contact manifolds $Y$ and $Y_{\Lambda}$ can be written as the union of $I \times Y$ (where $I$ is an interval) and a standard handle which is embedded in $\mathbb{R}^{2 n}$ and has the standard symplectic structure; see [20] for an explicit model of the handle and the attaching procedure. The product $I \times Y$ has a symplectic structure as part of the symplectization of $Y$. Using a normal form for neighborhoods of Legendrian submanifolds in contact 
manifolds, these two symplectic structures can be glued together on a neighborhood of the sphere where surgery takes place.

Let $\lambda$ be a $1-$ form with $\xi=\operatorname{ker}(\lambda)$. The Reeb vector field $R_{\lambda}$ of $\lambda$ is the unique vector field which satisfies $\lambda\left(R_{\lambda}\right)=1$ and $i_{R_{\lambda}} d \lambda=0$. For a generic $\lambda$, periodic Reeb orbits form a discrete set; see Bourgeois [2]. The algebra $\mathcal{A}(Y)$ of $Y$ is the supercommutative differential graded algebra (DGA) over the group ring $\mathcal{R}=\mathbb{Q}\left[H_{2}(Y ; \mathbb{Z})\right]$ freely generated by the good Reeb orbits (see [2]). The grading of a Reeb orbit is obtained by its Conley-Zehnder index (see Robbin and Salamon [18]) and the differential is defined by counting holomorphic curves in the symplectization of $Y$ (see [2]). The latter one is the manifold $\mathbb{R} \times Y$ equipped with the exact symplectic form $d\left(e^{t} \lambda\right), t \in \mathbb{R}$. The homology of the differential graded algebra $\mathcal{A}(Y)$ is the contact homology $\mathbb{H} C(Y, \xi)$ of $Y$.

Using the filtration of the algebra $\mathcal{A}(Y)$ by the length of its words, we look at $\mathcal{A}^{1}$ in $\mathcal{A}=\bigoplus_{l=0}^{\infty} \mathcal{A}^{l}$, ie we consider the submodule $\mathcal{A}^{1}$ of $\mathcal{A} / \mathcal{R}$ linearly generated by words of length 1. A DGA-homomorphism $\varepsilon: \mathcal{A} \rightarrow \mathcal{R}$, where $\mathcal{R}$ is equipped with the trivial differential, is called an augmentation. An augmentation $\varepsilon$ together with the differential $d$ on $\mathcal{A}(Y)$ induces a differential $d^{\varepsilon}$ on $\mathcal{A}(Y)$ which respects the filtration. The linearized contact homology $\mathbb{H} C^{\varepsilon}(Y, \xi)$ is the homology of $\mathcal{A}^{1}$ with the induced differential on $\mathcal{A}^{1}$.

Let $\Lambda$ be a Legendrian submanifold. A Reeb chord on $\Lambda$ is a flow line of $R_{\lambda}$ which begins and ends on $\Lambda$. For a generic $\lambda$, Reeb chords are isolated and the two endpoints of any Reeb chords are distinct. The algebra $\mathcal{A}(Y, \Lambda)$ of $\Lambda \subset Y$ is the noncommutative differential graded algebra generated by Reeb orbits and Reeb chords over the group ring $\mathbb{Q}\left[H_{2}(Y, \Lambda ; \mathbb{Z})\right]$. A Reeb chord is graded by its Conley-Zehnder index. For more details see Ekholm, Etnyre and Sullivan [9], where it is shown how one can close the path of Lagrangian subspaces of the contact planes along $\Lambda \subset \mathbb{R}^{2 n-1}$ by a positive rotation and define the Conley-Zehnder index for Reeb chords. The differential is defined by counting holomorphic curves in $\mathbb{R} \times Y$ with Lagrangian boundary condition $\mathbb{R} \times \Lambda$ (see McDuff and Salamon [16]). The homology of the differential graded algebra $\mathcal{A}(Y, \Lambda)$ is the Legendrian contact homology $L \mathbb{H} C(\Lambda)$ of $\Lambda$.

By restricting this Legendrian contact homology on Reeb chords, one corresponds a Legendrian homology algebra to a Legendrian submanifold. The corresponding algebra, $\operatorname{LHA}(\Lambda)$, carries the differential $d_{\mathrm{LHA}}: \operatorname{LHA}(\Lambda) \rightarrow \operatorname{LHA}(\Lambda)$ which satisfies the graded Leibniz rule

$$
d(a b)=(d a) b+(-1)^{|a|} a(d b),
$$

for arbitrary generators $a$ and $b$. 
From now on we consider $\Lambda$ as a union of Legendrian spheres. Then one can associate the following complexes to the differential graded algebra $\left(\operatorname{LHA}(\Lambda), d_{\mathrm{LHA}}\right)$ :

(1) A composable monomial in $\operatorname{LHA}(\Lambda)$ is a monomial $c_{1} \cdots c_{m}$ such that the origin of the chord $c_{i+1}$ lies on the same Legendrian sphere as the end of $c_{i}$ for $i=1, \ldots, m-1$. Let $\operatorname{LHO}(\Lambda) \subset \operatorname{LHA}(\Lambda)$ be linearly spanned by cyclically composable monomials, ie composable monomials $c_{1} \cdots c_{m}$ such that the end point of $c_{m}$ lies on the same Legendrian sphere as the origin of $c_{1}$. Since the differential $d_{\text {LHA }}$ of a cyclically composable monomial is the count of holomorphic discs in $\mathbb{R} \times \Lambda$, its differential is also in terms of cyclically composable monomials. We denote the restriction of the differential $d_{\mathrm{LHA}}$ to $\operatorname{LHO}(\Lambda)$ by $d_{\mathrm{LHO}}: \operatorname{LHO}(\Lambda) \rightarrow \operatorname{LHO}(\Lambda)$.

(2) Let $\mathrm{LHO}^{+}(\Lambda)$ be the module generated by nontrivial cyclically composable monomials of nonempty Reeb chords, ie $\mathrm{LHO}^{+}(\Lambda)=\operatorname{LHO}(\Lambda) /\langle 1\rangle$.

Let $P: \mathrm{LHO}^{+}(\Lambda) \rightarrow \mathrm{LHO}^{+}(\Lambda)$ be the linear map induced by the graded cyclic permutation

$$
P\left(c_{1} c_{2} \cdots c_{l}\right)=(-1)^{\left|c_{1}\right|\left(\left|c_{2}\right|+\cdots+\left|c_{l}\right|\right)} c_{2} \cdots c_{l} c_{1}
$$

for any $c_{1} \cdots c_{l} \in \mathrm{LHO}^{+}(\Lambda)$. Then $\mathrm{im}(\mathrm{Id}-P)$ is a subcomplex of $\mathrm{LHO}^{+}(\Lambda)$. So let us denote the quotient complex $\mathrm{LHO}^{+}(\Lambda) / \mathrm{im}(\mathrm{Id}-P)$ by $\mathrm{LH}^{\text {cyc }}(\Lambda)$. Let $d_{\text {cyc }}$ be the differential on $\mathrm{LH}^{\mathrm{cyc}}(\Lambda)$ induced by $d_{\mathrm{LHO}^{+}}$. Note that $\mathrm{LH}^{\mathrm{cyc}}(\Lambda)$ is not an algebra. It is a module generated by equivalence classes of cyclically composable monomials.

Let $w=c_{1} \cdots c_{m}$ be a monomial in $\operatorname{LHO}^{+}(\Lambda)$. We denote its image in $\operatorname{LH}^{\text {cyc }}(\Lambda)$ by $(w)$. A monomial $w \in \mathrm{LHO}^{+}(\Lambda)$ is called bad if, after acting a power of $P$ on it, it is the product of an even number of copies of an odd-graded monomial $w^{\prime}$. If a monomial is not bad it is called good.

Consider $w=a^{2 k}$ where $a \in \mathrm{LHO}^{+}(\Lambda)$ with the grading $|a|=2 l+1$. By applying $P$ on $a^{2 k}$ we have

$$
a^{2 k} \stackrel{\text { cyc }}{=}(-1)^{(2 l+1)((2 k-1)(2 l+1))} a^{2 k},
$$

ie $a^{2 k} \stackrel{\text { cyc }}{=}-a^{2 k}$ which means that $a^{2 k} \stackrel{\text { cyc }}{\equiv} 0$, or $\left(a^{2 k}\right)=0$. Hence, for any monomial $w \in \mathrm{LHO}^{+}(\Lambda)$, we have $(w)=0$ if and only if $w$ is bad. In other words, $\operatorname{LH}^{\text {cyc }}(\Lambda)$ is generated by the elements $(w)$, where $w$ is a good word in $\mathrm{LHO}^{+}$.

It is shown in [4] that $d_{\mathrm{cyc}}^{2}=0$ and the cyclic Legendrian homology, $L \mathbb{H}^{\mathrm{cyc}}(\Lambda)=$ $H_{*}\left(\mathrm{LH}^{\mathrm{cyc}}(\Lambda), d_{\mathrm{cyc}}\right)$, is independent of all choices and is a Legendrian isotopy invariant of $\Lambda$.

This work is motivated by [4], where the following surgery exact sequence is given: 
Theorem 1 (Bourgeois, Ekholm and Eliashberg [4]) There exists an exact sequence

$$
\cdots \rightarrow \mathbb{L} H_{k}^{\mathrm{cyc}}(\Lambda) \rightarrow \mathbb{H} C_{k}^{\Phi^{*} \varepsilon}\left(Y_{\Lambda}, \xi_{\Lambda}\right) \stackrel{\bar{\Phi}_{\varepsilon}}{\longrightarrow} \mathbb{H} C_{k}^{\varepsilon}(Y, \xi) \stackrel{\bar{\Psi}_{\varepsilon}}{\longrightarrow} L \mathbb{H}_{k-1}^{\mathrm{cyc}}(\Lambda) \rightarrow \cdots,
$$

where $\Phi$ is the chain map induced by the cobordism $\left(\overrightarrow{Y Y}_{\Lambda}, \omega\right)$ and $\Psi$ is a chain map induced by the symplectization $\left(\mathbb{R} \times Y, d\left(e^{t} \lambda\right)\right.$ ) with its Lagrangian submanifold $\mathbb{R} \times \Lambda$.

The geometric idea of the aforementioned linearization, using an augmentation $\varepsilon$, is to cap the punctures of the punctured holomorphic cylinders by holomorphic planes living in the symplectic filling (see [4]) of the contact manifold so that the differential counts the resulting capped holomorphic cylinders in terms of words of length one. In fact, in Theorem 1 the map $\bar{\Phi}_{\varepsilon}: \mathbb{H} C_{k}^{\Phi^{*} \varepsilon}\left(Y_{\Lambda}, \xi_{\Lambda}\right) \rightarrow \mathbb{H} C_{k}^{\varepsilon}(Y, \xi)$ counts rigid capped holomorphic curves in the symplectic cobordism $\left(\overrightarrow{Y Y}_{\Lambda}, \omega\right)$. On the other hand, the map $\bar{\Psi}_{\varepsilon}: \mathbb{H} C_{k}^{\varepsilon}(Y, \xi) \rightarrow \mathbb{H} C_{k-1}^{\text {cyc }}(\Lambda)$ counts rigid capped holomorphic curves in the symplectization $\left(\mathbb{R} \times Y, d\left(e^{t} \lambda\right)\right)$ with the boundary on $\mathbb{R} \times \Lambda$, which are asymptotic to a closed Reeb orbit at the positive end and to Reeb chords of $\Lambda$ at the negative end.

Let $\Lambda=\Lambda_{1} \sqcup \Lambda_{2}$ be a Legendrian link in the contact manifold $\left(Y_{0}, \xi_{0}\right)$. We would like to perform a Legendrian surgery along the link $\Lambda$ to obtain a new contact manifold $\left(Y_{2}, \xi_{2}\right)$. Then using the above surgery exact sequence, we will be able to compute the linearized contact homology of the new contact manifold $Y_{2}$. To this end, we need to know the cyclic Legendrian homology of $\Lambda$ denoted by $L \mathbb{H}^{\text {cyc }}(\Lambda)$. In general, the computations to obtain $L \mathbb{H}^{\text {cyc }}(\Lambda)$ are hard and long. In order to compute this invariant, we carry out the Legendrian surgery in two steps: first along $\Lambda_{1}$ in $Y_{0}$ and we call the obtained manifold $Y_{1}$, then along the second component called $\widetilde{\Lambda}_{2}$ in $Y_{1}$.

The goal of this work is to show that there exists a long exact sequence (Theorem 2)

$$
\cdots \rightarrow L \mathbb{H}_{k}^{\mathrm{cyc}}\left(\widetilde{\Lambda}_{2}\right) \rightarrow L \mathbb{H}_{k}^{\mathrm{cyc}}(\Lambda) \rightarrow L \mathbb{H}_{k}^{\mathrm{cyc}}\left(\Lambda_{1}\right) \rightarrow L \mathbb{H}_{k-1}^{\mathrm{cyc}}\left(\widetilde{\Lambda}_{2}\right) \rightarrow \cdots
$$

Then computing the cyclic Legendrian homology of $\Lambda$ can be simplified by computing the cyclic Legendrian homologies of $\Lambda_{1}$ and $\widetilde{\Lambda}_{2}$.

Knowing $L \mathbb{H}_{k}^{\mathrm{cyc}}\left(\tilde{\Lambda}_{2}\right)$ in fact provides two approaches for computing the linearized contact homology of the new contact manifold $Y_{2}$ obtained by the Legendrian surgery. Firstly, if we have $L \mathbb{H}_{k}^{\mathrm{cyc}}\left(\Lambda_{1}\right)$, then by using $L \mathbb{H}_{k}^{\mathrm{cyc}}\left(\tilde{\Lambda}_{2}\right)$ in the exact sequence of Theorem 2 we obtain $L \mathbb{H}_{k}^{\mathrm{cyc}}(\Lambda)$ and we use it in the surgery exact sequence of Theorem 1 to obtain the linearized contact homology of $Y_{2}$. Secondly, after the Legendrian surgery along $\Lambda_{1}$ we obtain the new contact manifold $Y_{1}$ containing $\tilde{\Lambda}_{2}$. We write the surgery exact sequence of Theorem 1 to obtain the linearized contact homology of $Y_{1}$. Performing surgery along $\widetilde{\Lambda}_{2}$ in $Y_{1}$, we obtain the contact manifold $Y_{2}$. Using again the surgery exact sequence of the Theorem 1 the linearized contact homology 
of $Y_{2}$ is obtained in terms of the cyclic Legendrian homology of $\tilde{\Lambda}_{2}$ and the linearized contact homology of $Y_{1}$.

Performing Legendrian surgery, we will see that the Reeb dynamics inside the attaching handle are described by generalized Dehn twist. Legendrian surgery along $\Lambda_{1}$ creates some chords on $\tilde{\Lambda}_{2}$. We show that there is a one-to-one correspondence between created chords on $\tilde{\Lambda}_{2}$ and cyclically composable words of chords on $\Lambda_{1} \sqcup \Lambda_{2}$, which start and end on $\Lambda_{2}$. We will denote such words by $c^{21} c_{1}^{1} \cdots c_{k}^{1} c^{12}, k \geq 0$. We decompose the module generated by the cyclic words of chords on $\Lambda$ into two modules $\operatorname{LH}^{\text {cyc }}\left(\Lambda_{1}\right)$ and $\mathrm{LH}^{\mathrm{cyc}}\left(\Lambda_{2}\right.$, mixed). Here $\mathrm{LH}^{\mathrm{cyc}}\left(\Lambda_{1}\right)$ is the module generated by the cyclic words containing the chords on $\Lambda_{1}$ and $\mathrm{LH}^{\mathrm{cyc}}\left(\Lambda_{2}\right.$, mixed) is the module generated by the cyclic words containing the chords which have at least one chord starting or ending on $\Lambda_{2}$. We show that $\operatorname{LH}$ cyc $\left(\tilde{\Lambda}_{2}\right)$ and $\operatorname{LH}^{\mathrm{cyc}}\left(\Lambda_{2}\right.$, mixed) are isomorphic modules. Then we define a chain map between these two modules and we show that this chain map is an isomorphism and we conclude with the long exact sequence for Legendrian links.

One can foresee abstract and computational applications for this exact sequence. In this paper we present some computational examples. We compute the cyclic Legendrian homology of the Legendrian link of simple Legendrian unknots. Then we compute the linearized contact homology of several lens spaces. Finally, using Morse flow trees techniques [8], we present our computations for Hopf link in higher dimensions.

\section{Towards the exact sequence}

\subsection{Cyclic Legendrian complexes}

Let $\Lambda=\Lambda_{1} \sqcup \Lambda_{2}$ be a Legendrian link in the contact manifold $\left(Y_{0}, \xi_{0}\right)$. And Let $\operatorname{LH}^{\text {cyc }}(\Lambda)$ be the module generated by good cyclic words in chords of $\Lambda$ over the group ring $\mathbb{Q}\left[H_{2}\left(Y_{0}, \Lambda ; \mathbb{Z}\right)\right]$. There are two types of cyclic words in chords of $\Lambda$ :

(1) Cyclic words of chords in $\Lambda_{1}$ : in this case, $\operatorname{LH}^{\text {cyc }}\left(\Lambda_{1}\right) \subset \operatorname{LH}^{\text {cyc }}(\Lambda)$ as a submodule.

(2) Cyclic words in which at least one chord starts or ends on $\Lambda_{2}$ : we denote the module generated by this type of cyclic words by $\operatorname{LH}^{\mathrm{cyc}}\left(\Lambda_{2}\right.$, mixed). In this case, we have $\operatorname{LH}^{\mathrm{cyc}}\left(\Lambda_{2}\right.$, mixed $) \subset \operatorname{LH}^{\text {cyc }}(\Lambda)$ as a submodule.

Notation We denote the chords that are in $\Lambda_{1}$ (and $\Lambda_{2}$ respectively) by $c^{1}$ (and $c^{2}$ respectively). We denote a chord from $\Lambda_{1}$ to $\Lambda_{2}$ by $c^{12}$ and a chord from $\Lambda_{2}$ to $\Lambda_{1}$ by $c^{21}$. 
Remark 1 The module $\operatorname{LH}^{\text {cyc }}\left(\Lambda_{1}\right)$ is not a subcomplex. For an arbitrary word $w=c_{1}^{1} \cdots c_{k}^{1} \in \operatorname{LH}^{\mathrm{cyc}}\left(\Lambda_{1}\right)$ the differential $d_{\text {cyc }} w$ is not necessarily in $\operatorname{LH}^{\mathrm{cyc}}\left(\Lambda_{1}\right)$. Using the Leibniz rule, the differential of $w$ is obtained by the differential of the chords $c_{i}^{1}$, $i=1, \ldots, k$. To compute the differential of $c_{i}^{1}$, when we leave the positive corner $c_{i}^{1}$ for the negative corners on the holomorphic disc we will meet mixed chords and chords which are in $\Lambda_{2}$. For instance, one can find $\partial_{\mathrm{LHA}} c_{i}^{1}=\cdots c^{12} c_{1}^{2} \cdots c_{l}^{2} c^{21}+\cdots, l \geq 0$, which is not in $\operatorname{LH}^{\mathrm{cyc}}\left(\Lambda_{1}\right)$.

Remark 2 The module $\operatorname{LH}^{\mathrm{cyc}}\left(\Lambda_{1}\right)$ is the quotient complex

$$
\operatorname{LH}^{\mathrm{cyc}}(\Lambda) / \mathrm{LH}^{\mathrm{cyc}}\left(\Lambda_{2}, \text { mixed }\right),
$$

that is, $\operatorname{LH}^{\text {cyc }}\left(\Lambda_{2}\right.$, mixed) is a subcomplex. An arbitrary word $w \in \operatorname{LH}^{\text {cyc }}\left(\Lambda_{2}\right.$, mixed $)$ can be of type $w=c_{1} \cdots c_{r} c_{1}^{2} c_{r+1} \cdots c_{k}$ or $w=c_{1} \cdots c_{r} c_{1}^{12} c_{r+1} \cdots c_{s} c_{1}^{21} c_{s+1} \cdots c_{k}$. Here the general notation for chords without superscript refers to the all possible chords so that $w$ is cyclically composable. For our purpose we have specified only one chord which is on $\Lambda_{2}$ or only two chords which end or start on $\Lambda_{2}$. Using the Leibniz rule, in the first case we have

$$
\begin{aligned}
d_{\mathrm{cyc}} w=\sum_{i=1}^{r} \pm c_{1} \cdots\left(\partial c_{i}\right) \cdots c_{r} c_{1}^{2} c_{r+1} \cdots c_{k} \pm & c_{1} \cdots c_{r}\left(\partial c_{1}^{2}\right) c_{r+1} \cdots c_{k} \\
& +\sum_{i=r+1}^{k} \pm c_{1} \cdots c_{r} c_{1}^{2} c_{r+1}\left(\partial c_{i}\right) \cdots c_{k}
\end{aligned}
$$

which is in $\operatorname{LH}^{\text {cyc }}\left(\Lambda_{2}\right.$, mixed). Again using the Leibniz rule, in the second case we have

$$
\begin{gathered}
d_{\mathrm{cyc}} w=\sum_{i=1}^{r} \pm c_{1} \cdots\left(\partial c_{i}\right) \cdots c_{r} c_{1}^{12} c_{s+1} \cdots c_{k} \pm c_{1} \cdots c_{r}\left(\partial c_{1}^{12}\right) c_{r+1} \cdots c_{r} c_{1}^{21} c_{s+1} \cdots c_{k} \\
+\sum_{i=r+1}^{s} \pm c_{1} \cdots c_{r} c_{1}^{12} c_{r+1}\left(\partial c_{i}\right) \cdots c_{s} c_{1}^{21} c_{s+1} \cdots c_{k} \\
\pm c_{1} \cdots c_{r} c_{1}^{12} c_{r+1} \cdots c_{s}\left(\partial c_{1}^{21}\right) c_{s+1} \cdots c_{k} \\
+\sum_{i=s+1}^{k} \pm c_{1} \cdots c_{r} c_{1}^{12} c_{r+1} \cdots c_{s} c_{1}^{21} c_{s+1}\left(\partial c_{i}\right) \cdots c_{k}
\end{gathered}
$$

which is in $\mathrm{LH}^{\text {cyc }}\left(\Lambda_{2}\right.$, mixed $)$.

By the above remark, we have the short exact sequence of complexes

$$
0 \rightarrow \operatorname{LH}^{\text {cyc }}\left(\Lambda_{2}, \text { mixed }\right) \rightarrow \operatorname{LH}^{\text {cyc }}(\Lambda) \rightarrow \operatorname{LH}^{\text {cyc }}\left(\Lambda_{1}\right) \rightarrow 0,
$$


which induces a long exact sequence in the level of homology:

(1) $\rightarrow L \mathbb{H}_{k}^{\mathrm{cyc}}\left(\Lambda_{2}\right.$, mixed $) \rightarrow L \mathbb{H}_{k}^{\mathrm{cyc}}(\Lambda) \rightarrow L \mathbb{H}_{k}^{\mathrm{cyc}}\left(\Lambda_{1}\right) \rightarrow L \mathbb{H}_{k-1}^{\mathrm{cyc}}\left(\Lambda_{2}\right.$, mixed $) \rightarrow$.

To reach our goal, we would like to know $L \mathbb{H}_{k}^{\mathrm{cyc}}\left(\Lambda_{2}\right.$, mixed) more precisely. The words in the module $\mathrm{LH}^{\mathrm{cyc}}\left(\Lambda_{2}\right.$, mixed) are of two types: cyclic words in chords of $\Lambda_{2}$ (which we denote by $\operatorname{LH}^{\mathrm{cyc}}\left(\Lambda_{2}\right)$ ) and the cyclic words that have at least one mixed chord (which we denote by $\operatorname{LH}^{\text {cyc }}($ mixed) $)$. Hence $\operatorname{LH}^{\text {cyc }}(\Lambda)=\operatorname{LH}^{\text {cyc }}\left(\Lambda_{1}\right) \oplus$ $\left(\mathrm{LH}^{\mathrm{cyc}}\left(\Lambda_{2}\right) \oplus \mathrm{LH}^{\mathrm{cyc}}(\right.$ mixed) $)$ and the differential is given by

$$
d_{\mathrm{cyc}}(\Lambda)=\left(\begin{array}{ccc}
d_{\mathrm{cyc}}\left(\Lambda_{1}\right) & 0 & 0 \\
0 & d_{\mathrm{cyc}}\left(\Lambda_{2}\right) & 0 \\
* & * & *
\end{array}\right) .
$$

\subsection{Effect of Legendrian surgery on Reeb dynamics}

Let $\left(Y_{0}, \lambda_{0}\right)$ be a contact manifold with dimension $2 n-1$. We assume that the Reeb flow isotopy $\phi_{R_{0}}^{t}\left(\Lambda_{1} \cup \Lambda_{2}\right)$, ie the time- $t$ flow of the Reeb vector field $R_{0}$, intersects $\Lambda_{1} \cup \Lambda_{2}$ transversely. Consider the contact manifold $\left(Y_{1}, \lambda_{1}\right)$ which is obtained by surgery on $\Lambda_{1} \subset Y_{0}$. The Reeb vector field $R_{1}$ on $Y_{1}$ satisfies $R_{0}=R_{1}$ outside a tubular neighborhood $U$ of $\Lambda_{1} \subset Y_{0}$. Under surgery, $U$ is replaced by the unit cotangent bundle of the $n$-disc which is the Lagrangian core disc of the attached handle. The Reeb dynamics inside the handle is the geodesic flow of the flat disc; see Geiges [14, Theorem 1.5.2].

Let $J^{1}\left(S^{n-1}\right) \approx T^{*} S^{n-1} \times \mathbb{R}$ be the 1 -jet space of the $(n-1)$-sphere with its standard contact form $\alpha=d z+p d q$, where $(q, p)$ are canonical coordinates on $T^{*} S^{n-1}$ and $z$ is a coordinate in the $\mathbb{R}$-factor. Let $S \subset J^{1}\left(S^{n-1}\right)$ denote the 0 -section and define

$$
U_{\varepsilon}=\{|z| \leq \varepsilon,\|p\| \leq \varepsilon\}, \quad \varepsilon>0,
$$

where $S$ is considered as the unit sphere in $\mathbb{R}^{n}$ in order to define $\|p\|$. So $U_{\varepsilon}$ is a neighborhood of $S$ of size $\varepsilon$. Let $V_{ \pm}=\{z= \pm \varepsilon\}$. The form $\omega_{ \pm}=\left.d \alpha\right|_{V_{ \pm}}$is symplectic and we will see in Lemma 1 that $\left(V_{ \pm}, \omega_{ \pm}\right)$is symplectomorphic to the cotangent disc bundle of $S^{n-1}$ of radius $\varepsilon$. By the Legendrian neighborhood theorem, for $\varepsilon>0$ sufficiently small, there exists an embedding $\boldsymbol{j}: U_{\varepsilon} \rightarrow Y_{0}$ with $\boldsymbol{j}^{*} \lambda_{0}=\alpha$ and $\boldsymbol{j}(S)=\Lambda_{1}$, which allows us to identify $U_{\varepsilon}$ with a neighborhood of $\Lambda_{1} \subset Y_{0}$. We keep the notation $U_{\varepsilon}$ and $V_{ \pm}$for the images of $U_{\varepsilon}$ and $V_{ \pm}$, respectively, under the contact embedding $\boldsymbol{j}$. The Reeb flow of $\lambda_{1}$ defines a symplectomorphism

$$
i:\left(V_{-},\left.d \lambda_{0}\right|_{V_{-}}\right) \rightarrow\left(V_{+},\left.d \lambda_{0}\right|_{V_{+}}\right) .
$$


The effect of the surgery on Reeb dynamics is as follows. Outside $U_{\varepsilon}$ we have $R_{1}=R_{0}$. All the trajectories which enter $U_{\varepsilon}$ through $V_{-}$exit through $V_{+}$and thus there is a symplectomorphism

$$
\tilde{\tau}:\left(V_{-},\left.d \lambda_{0}\right|_{V_{-}}\right) \rightarrow\left(V_{+},\left.d \lambda_{0}\right|_{V_{+}}\right)
$$

In fact $\tilde{\tau}=\tau \circ \boldsymbol{i}$ in which $\tau:\left(V_{+},\left.d \lambda_{0}\right|_{V_{+}}\right) \rightarrow\left(V_{+},\left.d \lambda_{0}\right|_{V_{+}}\right)$is a generalized symplectic Dehn twist where we identify $\left(V_{+},\left.d \lambda_{0}\right|_{V_{+}}\right)$with the cotangent disc bundle of the sphere $S^{n-1}$ of radius $\varepsilon$. In the following we recall the definition of the generalized Dehn twist. Before that let us fix some notation.

Notation We consider $\mathbb{R}^{2 n} \approx \mathbb{C}^{n}$ with coordinates $(u, v)=u+i v, u, v \in \mathbb{R}^{n}$. If $u=\left(u_{1}, \ldots, u_{n}\right)$ are coordinates on $\mathbb{R}^{n}$ then we consider $d u=\left(d u_{1}, \ldots, d u_{n}\right) \in$ $\left(\left(\mathbb{R}^{n}\right)^{*}\right)^{n}$ as a vector of cotangent vectors, and $\partial_{u}=\left(\partial_{u_{1}}, \ldots, \partial_{u_{n}}\right) \in\left(\mathbb{R}^{n}\right)^{n}$ as a vector of tangent vectors. If $u, v \in \mathbb{R}^{n}$ then we write the standard metric $g_{\mathrm{st}}(u, v)=u \cdot v=$ $\sum_{j=1}^{n} u_{j} v_{j}$ and we use the abbreviation $u^{2}=u \cdot u$. Let also $\|u\|=\sqrt{u^{2}}$.

Definition 1 (Generalized Dehn twist [19]) Consider the cotangent bundle of $S^{n-1}$,

$$
T^{*} S^{n-1}=\left\{(u, v) \in \mathbb{R}^{n} \times \mathbb{R}^{n} \mid u . v=0,\|u\|=1\right\}
$$

(under the identification $T S^{n-1} \cong T^{*} S^{n-1},(u, v) \mapsto\left(u, v^{*}\right)$ where $v^{*}$ is dual to $v$ under $\left.g_{\text {st }}\right)$ with the standard symplectic form $\omega_{T^{*} S^{n-1}}$. Let $S$ be the zero section. The length function $\mu: T^{*} S^{n-1} \rightarrow \mathbb{R}$ given by $\mu(u, v)=\|v\|$ generates a Hamiltonian circle action on $T^{*} S^{n-1} \backslash S$ whose flow is given by

$$
\varphi_{t}^{\mu}(u, v)=\left(\cos (t) u+\sin (t) \frac{v}{\|v\|}, \cos (t) v-\sin (t)\|v\| u\right) .
$$

The time- $\pi$ map $\varphi_{\pi}^{\mu}$ extends over the zero section by the antipodal map $A(u, 0)=$ $(-u, 0)$. Let $R: \mathbb{R} \rightarrow \mathbb{R}$ be a smooth function such that $R(t)=0$ for $t \gg 0$ and $R(-t)=R(t)-t$ for small $|t|$. We can think of the function $R$ as a bump function with $\operatorname{supp}(R) \subset(-\infty,|t|)$ for small $|t|$. Let $H=R \circ \mu$. The flow of $H$ is $\varphi_{t}^{H}(u, v)=$ $\varphi_{t R^{\prime}(\|v\|)}^{\mu}(u, v)$. Since $R^{\prime}(0)=\frac{1}{2}, \varphi_{2 \pi}^{H}$ extends continuously to $T^{*} S^{n-1}$ by the antipodal map. By [19] this extension is smooth and hence it is a symplectomorphism. Now let $\sigma$ be the time- $2 \pi$ flow of $H=R \circ \mu$ on $T^{*} S^{n-1} \backslash S$ as above, extended to $S$ by the antipodal map. The symplectomorphism $\sigma$ is called the generalized Dehn twist.

Remark 3 We recall the definition of the Hamiltonian circle action. A symplectic circle action on a symplectic manifold $(M, \omega)$ is a smooth family of symplectomorphisms $\psi_{t} \in \operatorname{Symp}(M, \omega), t \in S^{1}$, such that $\psi_{t+s}=\psi_{t} \circ \psi_{s}$ for any $t, s \in S^{1}$. One 
can easily check that the corresponding vector fields $X_{t} \equiv \frac{d}{d t} \psi_{t} \circ \psi_{t}^{-1}$ are timeindependent, ie $X_{t}=X$ is constant in $t$. We call $X$ the associated vector field of the given symplectic circle action.

Since $\psi_{t}$ is a symplectomorphism for all $t, L_{X} \omega=0$, and hence by Cartan's formula, $d\left(i_{X} \omega\right)=0$. This means that $X$ is a symplectic vector field. When $i_{X} \omega=d H$ is an exact 1 -form, the corresponding symplectic circle action is called a Hamiltonian circle action.

Now consider $\left(\mathbb{R}^{2 n}, \omega_{\mathrm{st}}=\sum_{j=1}^{n} d u_{j} \wedge d v_{j}\right)$ with the symplectic circle action given by the complex multiplication

$$
\begin{aligned}
(u, v)=\left(u_{1}+i v_{1}, \ldots,\right. & \left.u_{n}+i v_{n}\right) \\
& \mapsto\left(e^{i t}\left(u_{1}+i v_{1}\right), \ldots, e^{i t}\left(u_{n}+i v_{n}\right)\right)=e^{i t}(u, v), \quad t \in S^{1} .
\end{aligned}
$$

Note that the circle action is generated by the vector field $X=\sum_{j=1}^{n}\left(-v_{j} \partial_{u_{j}}+u_{j} \partial_{v_{j}}\right)$. Therefore the Hamiltonian function $H$ defined by $d H=-i_{X} \omega_{\text {st }}$ is given by $H(u, v)=$ $-\frac{1}{2}|(u, v)|^{2}$.

Lemma 1 The map $\tau$ is a generalized Dehn twist.

Proof Consider $S T^{*} D^{n}$ as the unit cotangent bundle over the unit disc. Let $(x, y) \in V_{-}$ be the point through which we enter to the handle, where $x$ is a point on disc and $y$ is the direction by which we enter the handle. Also let $\left(x^{\prime}, y^{\prime}\right) \in V_{+}$be the point through which we exit the handle, where $x^{\prime}$ is a point on the disc and $y^{\prime}$ is the direction by which we exit the handle. Since the Reeb trajectories on the flat disc are straight lines, the directions $y$ and $y^{\prime}$ are the same.

Now consider the tangent plane to the disc at the point $(x, y)$. We can split $y$ into the sum of the normal vector and the tangent vector at this point, ie $y=y_{N}+y_{T}$. As $\|y\|=1$, we obtain $y_{N}$ in a unique way. So we can consider $y$ in correspondence to $y_{T}$. Hence the map

$$
f: V_{-} \rightarrow T^{*} S^{n-1}, \quad(x, y) \mapsto\left(x, y_{T}\right),
$$

identifies $V_{-}$with the cotangent disc bundle of the sphere $S^{n-1}$ of radius $\varepsilon$. In the same way, using the map

$$
g: V_{+} \rightarrow T^{*} S^{n-1}, \quad\left(x^{\prime}, y^{\prime}\right) \mapsto\left(x^{\prime}, y_{T}^{\prime}\right),
$$




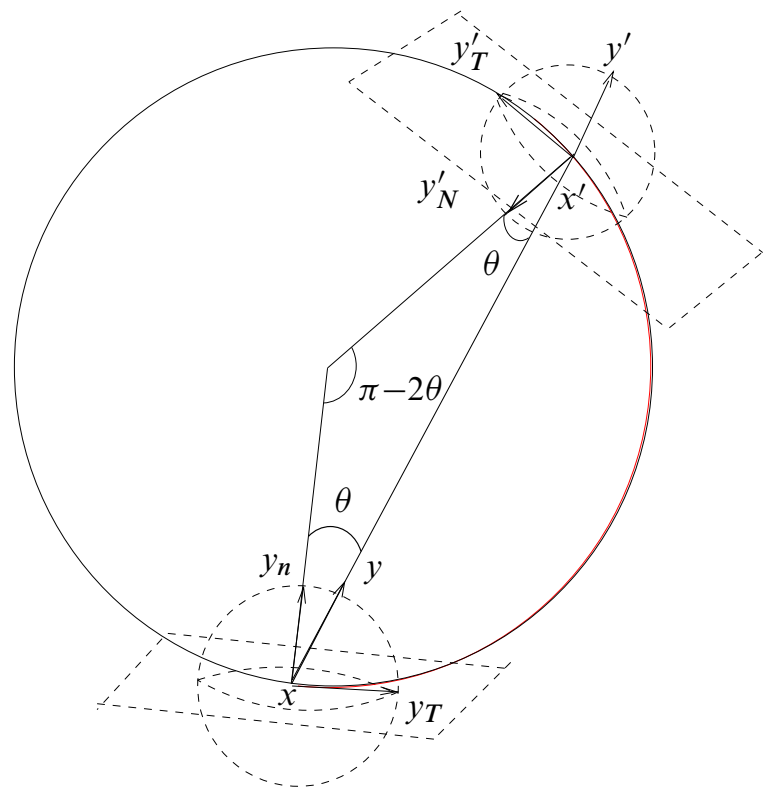

Figure 1: Dehn twist lemma

we identify $V_{+}$with the cotangent disc bundle of the sphere $S^{n-1}$ of radius $\varepsilon$. Moreover the map $g^{-1} \circ f$ gives the identification map $i: V_{-} \rightarrow V_{+}$.

Note that the arc (the red arc in Figure 1) between the points $(u, v):=\left(x, y_{T}\right) \in$ $T^{*} S^{n-1}$ and $\left(u^{\prime}, v^{\prime}\right):=\left(x^{\prime}, y_{T}^{\prime}\right) \in T^{*} S^{n-1}$ is a part of the flow trajectory on the sphere $S^{n-1}$. On the other hand, the Reeb flow on $S T^{*} S^{n-1}$ is the same as the geodesic flow on $S^{n-1}$; see [14, Theorem 1.5.2]. Therefore, we have $\left(u^{\prime}, v^{\prime}\right)=$ $\varphi_{\pi-2 \theta}^{R_{\lambda}}(u, v)$, where $0 \leqslant \theta \leqslant \frac{\pi}{2}$ is the angle between $y$ and $y_{N}$. In fact the intersection of the plane $y \wedge y_{N}$ with the ball $B^{n}$ provides the disc $D^{2}$, and the flow time $\pi-2 \theta$ is the central angle which corresponds to the third angle of the isosceles triangle in $D^{2}$. We define the map $\tau: V_{+} \rightarrow V_{+}$(under the identification map $\boldsymbol{i}$ ) by $\tau(u, v)=\varphi_{\pi-2 \theta}^{R_{\lambda}}(u, v)$, with $0 \leqslant \pi-2 \theta \leqslant \pi$. Clearly, $\tau$ is a generalized Dehn twist, since the Reeb flow $\varphi_{t}^{R_{\lambda}}$ is the same as the Hamiltonian flow of the length function $\mu$ on $T^{*} S^{n-1}$ (by [14, Theorem 1.5.2]). Moreover $\tau$ is the time- $2 \pi$ flow of the Hamiltonian function $H=R \circ \mu$ on $T^{*} S^{n-1}$, where $R$ is a suitable function with the description in Definition 1, such that $2 \pi R^{\prime}(\|v\|)=\pi-2 \theta$. At the time zero, ie when $\theta=\frac{\pi}{2}$, we are on the boundary of $V_{-}$so the entering point and the exiting point are the same. Hence the map $\tau$ is the identity. At time $\pi$, ie when $\theta=0$, we are on the center of $V_{-}$, and the exiting point is the antipode of the entering point. Therefore the map $\tau$ is the antipodal map. 
Below we illustrate the map $\tilde{\tau}$ in dimension $2 n-2=2$. Assume that the Reeb trajectory enters into the handle vertically through $V_{-}$at the point $a_{1}$. Then it will leave the flat disc at the antipodal point of $a_{1}$ through $V_{+}$. In Figure 2, we show this antipodal point by $\tilde{a}_{1}$. Now assume that the Reeb trajectory enters into the handle tangently at the point $a_{2}$. In this case, as the Reeb trajectory enters through $V_{-}$it exits immediately at $a_{2}$ through $V_{+}$. Similarly, for the Reeb trajectory that enters into the handle at the point $a_{3}$, tangently but in the opposite direction, we show the exiting point by $a_{3}$. Finally, let the Reeb trajectory enter into the handle at $a_{4}$ through $V_{-}$with an angle between the vertical and tangent directions. Then it exits the handle through $V_{+}$at a point between $a_{4}$ and its antipodal point. We show this exiting point by $\tilde{a}_{4}$.

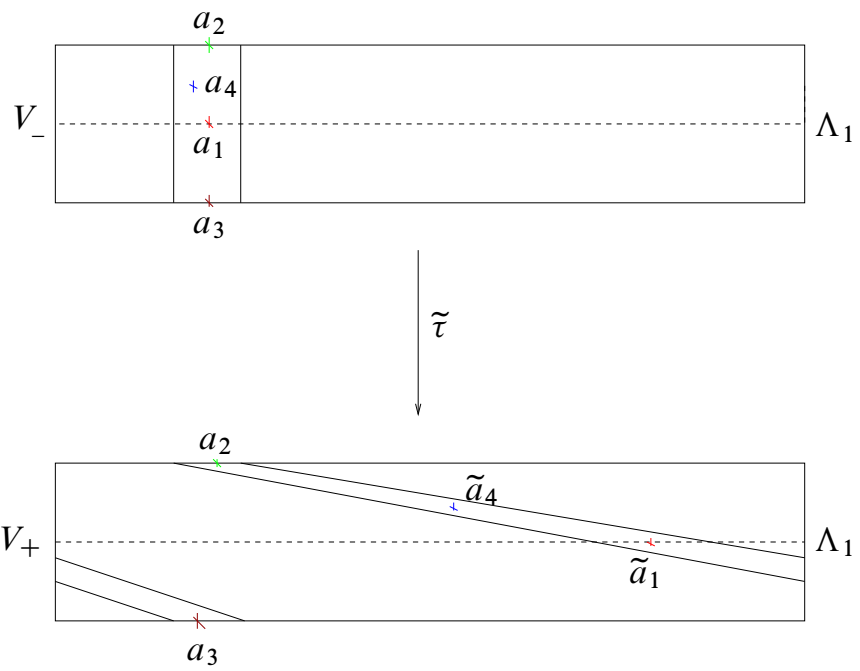

Figure 2: The map $\tilde{\tau}$ in dimension $2 n-2=2$

After Legendrian surgery along $\Lambda_{1}$, there are in fact two types of Reeb chords for $\tilde{\Lambda}_{2}$. Given any $C>0$ we can find a sufficiently small $\varepsilon>0$ (the size of the surgery region) such that all chords on $\Lambda_{2}$ (denoted $\mathcal{C}\left(\Lambda_{2}\right)$ ) of length less than $C$ survive the surgery.

On the other hand, the mixed chords on $\Lambda_{1} \sqcup \Lambda_{2}$ which get close to the surgery region will create new Reeb chords for $\tilde{\Lambda}_{2}$ by the surgery. In the following we show that these created chords are in one-to-one correspondence to the words of the type $c_{1}^{21} c_{1}^{1} \cdots c_{k}^{1} c_{1}^{12}, k \geq 1$. Here $c_{k}^{1}$ denotes the empty letter when we have $c^{21} c^{12}$ or a chord on $\Lambda_{1}$.

Proposition 1 By the Legendrian surgery along $\Lambda_{1}$ there exists a one-to-one correspondence between the created chords $\widetilde{c}_{k}$ in $\mathcal{C}\left(\tilde{\Lambda}_{2}\right)$ and the words $c_{1}^{21} c_{1}^{1} \cdots c_{k}^{1} c_{1}^{12}$ $(k \geq 1)$ of chords in $\mathcal{C}\left(\Lambda_{1} \sqcup \Lambda_{2}\right)$. 
Proof We start with the case $c^{21} c^{12}$. Let $c^{21}$ be the chord from $\Lambda_{2}$ to $\Lambda_{1}$ which is entering into the attached handle through $V_{-}$. For $\delta>0$, we denote the Reeb flow in the $\delta$-neighborhood of $c^{21}$ by $\phi_{21}$. By assumption we know that $\phi_{21_{*}}\left(T \Lambda_{2}\right) \pitchfork T \Lambda_{1}$, ie $c^{21}$ is nondegenerate. Consider the tangent space $T \Lambda_{2}$ at the starting point of $c^{21}$, and the Reeb trajectory $\tilde{c}$ in the $\delta$-neighborhood of $c^{21}$. The chord $\tilde{c}$ starts near the starting point of $c^{21}$ and enters into the handle through $V_{-}$at a point near the entering point of $c^{21}$ into the handle. Choosing $\varepsilon>0$ sufficiently small, we shrink $V_{-}$in the $D^{n-1}$ direction (vertically in Figure 3 , when $2 n-2=2$,) so that this point is getting very close to $\Lambda_{1}$. By the transversality assumption, $\phi_{21_{*}}\left(T \Lambda_{2}\right)$ will be in the $D^{n-1}$ direction (vertical to $\Lambda_{1}$ in Figure 3 ). When we enter into the attached handle, by Lemma 1 , the Reeb flow defines a symplectomorphism $\tilde{\tau}$ by a generalized Dehn twist. The image of $\phi_{21_{*}}$ under the map $\tilde{\tau}$ will be in the $S^{n-1}$ direction. Again by choosing sufficiently small $\varepsilon>0$ we shrink $V_{+}$so that $\tilde{\tau}\left(\phi_{21_{*}}\left(T \Lambda_{2}\right)\right)$ gets close enough to $S^{n-1} \times\{0\}$ (to the horizontal line in Figure 3), that is to $\Lambda_{1}$. Next we exit the attached handle through $V_{+}$and we continue our journey in the $\delta$-neighborhood of the chord $c^{12}$. The chord $\tilde{c}$ ends at $\Lambda_{2}$ very close to the end point of the chord $c^{12}$. By the transversality assumption we have $\phi_{12_{*}}^{-1}\left(T \Lambda_{2}\right) \pitchfork T \Lambda_{1}$. Again by shrinking $V_{+}$, $\phi_{12_{*}}^{-1}\left(T \Lambda_{2}\right)$ is in $D^{n-1}$ direction (vertical in Figure 3 ) and hence it is vertical to the trajectory in the $S^{n-1}$ direction which is leaving the attached handle through $V_{+}$. This vertical intersection shows that there is a unique end point for the chord $\tilde{c}$. In other words the chord $\tilde{c}$ is constructed uniquely near the union of chords $c^{21}$ and $c^{12}$.

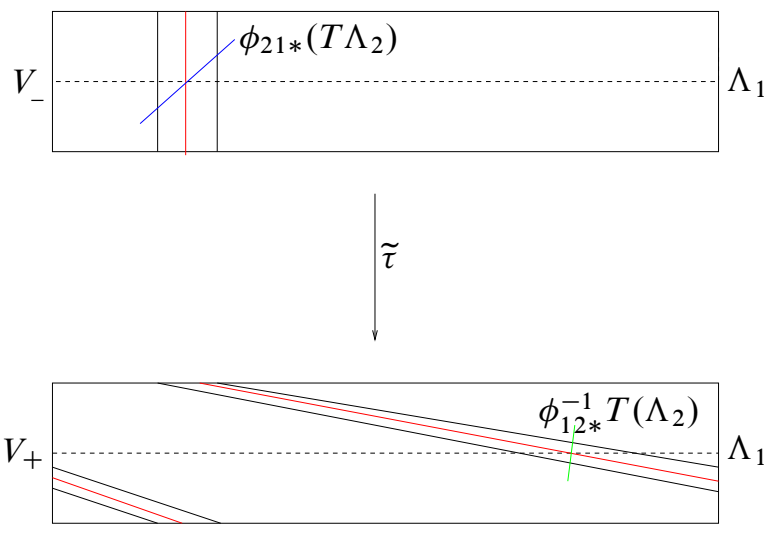

Figure 3: Effect of Legendrian surgery in dimension $2 n-2=2$

Now consider the general case $c^{21} c_{1}^{1} \cdots c_{k}^{1} c^{12}$. We repeat the argument in the above case until the Reeb flow exits the attached handle through $V_{+}$. The Reeb trajectory is almost in the $S^{n-1}$ direction (the horizontal direction in Figure 3). We continue our journey in the $\delta$-neighborhood of the chord $c_{1}^{1}$. The chord $\tilde{c}$ gets to $\Lambda_{1}$ near the end 
point of the chord $c_{1}^{1}$. Since $\Lambda_{1}$ is Legendrian, the Reeb trajectory is transverse to $\Lambda_{1}$. So its translation to $V_{+}$is exactly matched with the $D^{n-1}$ direction (by shrinking $V_{+}$). Hence it is vertical to the trajectory which is leaving the attached handle through $V_{+}$. This vertical intersection shows that there is a unique end point for the chord $\tilde{c}$ getting to $\Lambda_{1}$. Now again we are entering the attached handle through $V_{-}$with the Reeb trajectory which is exactly in the $D^{n-1}$ direction. The same as what we explained above, the symplectomorphism $\tilde{\tau}$ twists the trajectories in $D^{n-1}$ direction (the vertical direction in Figure 3) to the trajectories in the $S^{n-1}$ direction (the horizontal direction in Figure 3) which is now matched with $\Lambda_{1}$. If we continue our journey in the $\delta-$ neighborhood of the chord $c_{1}^{1}$, or $c_{2}^{1}, \ldots, c_{k}^{1}$, we repeat the same argument. In fact whenever we enter the attached handle the symplectomorphism $\tilde{\tau}_{i}, i=1, \ldots, k$, twists the flow lines. Therefore the intersection is always vertical and we obtain the unique end and hence the unique chord. Finally, we continue our journey in the $\delta$-neighborhood of the chord $c^{12}$. The chord $\tilde{c}$ gets to $\Lambda_{2}$ near the end point of the chord $c^{12}$. By the transversality assumption we have $\phi_{12_{*}}^{-1}\left(T \Lambda_{2}\right) \pitchfork T \Lambda_{1}$. Again by shrinking $V_{+}$, $\phi_{12_{*}}^{-1}\left(T \Lambda_{2}\right)$ is the vertical trajectory. Hence it is vertical to the trajectory which leaves the attached handle through $V_{+}$. This vertical intersection shows that there is a unique end point for the chord $\tilde{c}$ getting to $\Lambda_{2}$.

From the above explanation we conclude that there is an injective map from the set $\left\{c^{21} c_{1}^{1} \cdots c_{k}^{1} c^{12} \mid k \geq 1\right\}$ to the set $\left\{\tilde{c}_{k} \mid k \geq 1\right\}$ which assigns to each $c^{21} c_{1}^{1} \cdots c_{k}^{1} c^{12}$ a unique $\tilde{c}_{k}$. Note that we need to choose $\delta>0$ small enough so that $\delta$-neighborhoods of any two elements $c^{21} c_{1}^{1} \cdots c_{k}^{1} c^{12}$ and $c^{21} c_{1}^{1} \cdots c_{l}^{1} c^{12}, k \neq l$, are disjoint. Next we show that this map is surjective.

Let $\tilde{c}$ be any Reeb chord of $\tilde{\Lambda}_{2}$ created by the surgery along $\Lambda_{1} \subset Y_{0}$. The chord $\tilde{c}$ contains the trajectory $c$ in $Y_{1} \backslash U_{\varepsilon}$ from $V_{+}$to $V_{-}$. We want to show that the portion $c$ of $\tilde{c}$ is close to some chords on $\Lambda_{1} \sqcup \Lambda_{2}$. That is for each $\delta>0$, there exists $\varepsilon>0$ such that $c$ is contained in a $\delta$-neighborhood of some chords on $\Lambda_{1} \sqcup \Lambda_{2}$. By contradiction assume that there exists $\delta>0$, such that for each $\varepsilon>0$, the chord $c$ does not stay in a $\delta$-neighborhood of any chord of $\Lambda_{1} \sqcup \Lambda_{2}$. For $\varepsilon=\frac{1}{n}$, denote the Reeb trajectory by $c_{n}$ (from $V_{+}$to $V_{-}$). Let $a_{n} \in V_{+}$be the starting point of $c_{n}$. Since $V_{-}$and $V_{+}$ are compact we extract a subsequence so that when $a_{i_{n}}$ converge to $a \in V_{+}$, the Reeb flows $\varphi_{R}^{T_{i_{n}}}\left(a_{i_{n}}\right) \in V_{-}$converge to $\varphi_{R}^{T}(a) \in V_{-}$when $i_{n} \rightarrow \infty$ for $T_{i_{n}}=\operatorname{length}\left(c_{i_{n}}\right)$. Therefore there exists a chord of $\Lambda_{1} \sqcup \Lambda_{2}$ starting at $a$. When $a_{n} \rightarrow a$, then $c_{n} \rightarrow c$ for $n$ large enough. Therefore $c_{n}$ is contained in a $\delta$-neighborhood of a chord of $\Lambda_{1} \sqcup \Lambda_{2}$.

As long as the created Reeb chord $\tilde{c}$ enters into the attached handle, for each portion of $\tilde{c}$ outside of the handle we repeat the same argument. Hence there is a union of chords of $\Lambda_{1} \sqcup \Lambda_{2}$ so that $\tilde{c}$ is in a $\delta$-neighborhood of them. 


\subsection{Constructing a chain map}

Let $\mathrm{LH}^{\text {cyc }}\left(\widetilde{\Lambda}_{2}\right)$ be the module generated by cyclic words in chords of $\tilde{\Lambda}_{2} \subset Y_{1}$. Chords of $\widetilde{\Lambda}_{2}$ have two types: the chords of $\Lambda_{2}$ which are unaffected by surgery along $\Lambda_{1}$, or the chords created by the surgery which are in a one-to-one correspondence with the words of the type $c_{1}^{21} c_{1}^{1} \cdots c_{k}^{1} c_{1}^{12}$.

Proposition 2 We have $\operatorname{LH}^{\mathrm{cyc}}\left(\widetilde{\Lambda}_{2}\right) \cong \operatorname{LH}^{\mathrm{cyc}}\left(\Lambda_{2}\right.$, mixed) as modules.

Proof Let $w=\widetilde{c}_{1} \cdots \widetilde{c}_{m}$ be a cyclic word in $\operatorname{LH}^{\text {cyc }}\left(\widetilde{\Lambda}_{2}\right)$, where the $\widetilde{c}_{i}(i=1, \ldots, m)$ are chords of $\tilde{\Lambda}_{2}$. By the previous proposition each $\widetilde{c}_{i}$ is in one-to-one correspondence with a chord $c^{2}$ in $\operatorname{LH}^{\text {cyc }}\left(\Lambda_{2}\right)$ or with $c_{1}^{21} c_{1}^{1} \cdots c_{i}^{1} c_{1}^{12}$ in $\operatorname{LH}^{\text {cyc }}\left(\Lambda_{2}\right.$, mixed $)$. We define an algebra map

$$
h: \operatorname{LH}^{\mathrm{cyc}}\left(\widetilde{\Lambda}_{2}\right) \rightarrow \operatorname{LH}^{\mathrm{cyc}}\left(\Lambda_{2}, \text { mixed }\right)
$$

by $h\left(\tilde{c}_{i}\right)=c^{2}$, where $\tilde{c}_{i}$ is a chord of type one, and $h\left(\tilde{c}_{i}\right)=c_{1}^{21} c_{1}^{1} \cdots c_{i}^{1} c_{1}^{12}$, where $\tilde{c}_{i}$ is a chord of type two. The right-hand sides are not in $\operatorname{LH}^{\mathrm{cyc}}\left(\Lambda_{1}\right)$, so they are in $\mathrm{LH}^{\text {cyc }}\left(\Lambda_{2}\right.$, mixed). To show this map is an isomorphism we define an inverse map as follows. In an arbitrary cyclic word $w$ in $\operatorname{LH}^{\text {cyc }}\left(\Lambda_{2}\right.$, mixed) we insert a subdivision "|" before and after $c^{2}$, before $c^{21}$, and after $c^{12}$. Then we define an algebra map

$$
g: \operatorname{LH}^{\text {cyc }}\left(\Lambda_{2}, \text { mixed }\right) \rightarrow \operatorname{LH}^{\text {cyc }}\left(\widetilde{\Lambda}_{2}\right)
$$

by $g\left(\left|c^{2}\right|\right)=c^{2}$ and $g\left(\left|c_{1}^{21} c_{1}^{1} \cdots c_{i}^{1} c_{1}^{12}\right|\right)=\tilde{c}_{i}$. Hence we conclude that the map $g$ is the inverse of the map $h$.

In order to prove that the modules $\operatorname{LH}^{\text {cyc }}\left(\widetilde{\Lambda}_{2}\right)$ and $\operatorname{LH}^{\text {cyc }}\left(\Lambda_{2}\right.$, mixed) have isomorphic cyclic Legendrian homologies, ie $L \mathbb{H}^{\text {cyc }}\left(\tilde{\Lambda}_{2}\right) \cong L \mathbb{H}^{\text {cyc }}\left(\Lambda_{2}\right.$, mixed $)$, we define a chain map $\Phi: \operatorname{LH}^{\text {cyc }}\left(\widetilde{\Lambda}_{2}\right) \rightarrow \operatorname{LH}^{\text {cyc }}\left(\Lambda_{2}\right.$, mixed $)$ that counts holomorphic discs in the surgery cobordism $W_{1}$ between $Y_{0}$ and $Y_{1}$.

Let $L_{1}$ be the Lagrangian core disc of the attached handle, $\left(L_{1}, \partial L_{1}\right) \subset\left(W_{1}, \partial W_{1}\right)$, which intersects $\partial_{-} W_{1}=Y_{0}$ along the Legendrian submanifold $\Lambda_{1}=L_{1} \cap \partial_{-} W_{1}$ as shown in Figure 4. Also let the vertical cylinder $L_{2}$ be an exact Lagrangian submanifold $\left(L_{2}, \partial L_{2}\right) \subset\left(W_{1}, \partial W_{1}\right)$ which intersects $\partial_{+} W_{1}=Y_{1}$ and $\partial_{-} W_{1}=Y_{0}$ along the Legendrian submanifolds $\widetilde{\Lambda}_{2}=L_{2} \cap \partial_{+} W_{1}$ and $\Lambda_{2}=L_{2} \cap \partial_{-} W_{1}$.

Consider unit disc $D$ with conformal structure $j$ and with the boundary punctures $z_{+}, z_{1-}, \ldots, z_{k-}$. A map $f:\left(D, \partial D \backslash\left\{z_{+}, z_{1-}, \ldots, z_{k-}\right\}\right) \rightarrow\left(W_{1}, L\right)$ is holomorphic if $d f \circ j=J \circ d f$, where $J$ is almost complex structure on $W_{1}$ and $L=L_{1} \cup L_{2}$ is the Lagrangian boundary condition satisfied by $f$. We say that $f$ is asymptotic to the chord $\tilde{c}$ in the collection of the chords $\mathcal{C}\left(\widetilde{\Lambda}_{2}\right)$ of length $T_{+}$at the boundary puncture $z_{+}$at $+\infty$ if: 
(1) $f$ maps a pointed neighborhood $U_{+}$of $z_{+}$into $[0,+\infty) \times Y_{1}$, so that $f(z)=$ $(a(z), u(z))$ for all $z \in U_{+}$.

(2) $\lim _{z \rightarrow z_{+}} a(z)=+\infty$.

(3) In holomorphic polar coordinates $(\rho, \theta), \theta \in[-\pi, 0]$, centered at $z_{+}$(where $\theta \in\{-\pi, 0\}$ along $\partial \Sigma)$, we have $\lim _{\rho \rightarrow 0} u(\rho, \theta)=\tilde{c}\left(-\left(T_{+} / \pi\right) \theta\right)$.

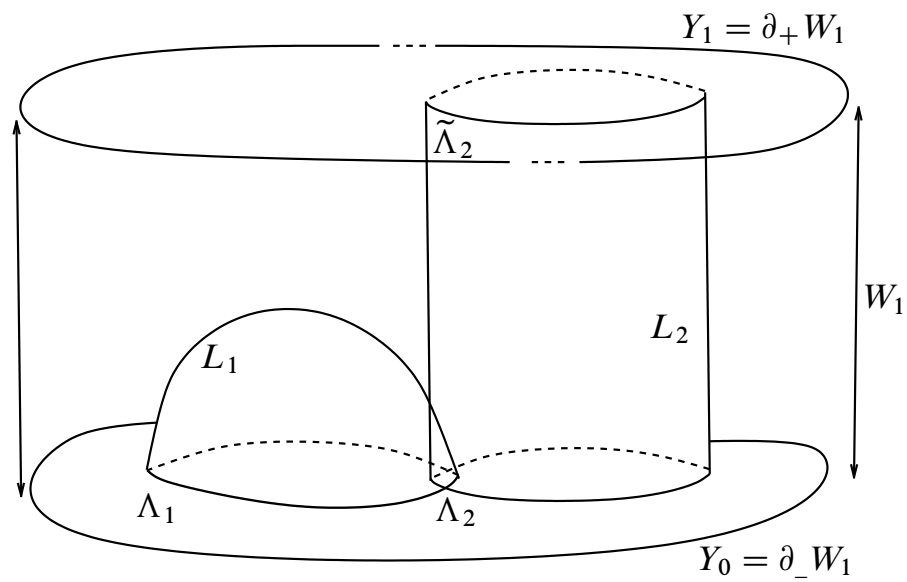

Figure 4: Surgery cobordism $W_{1}$

Let $\mathcal{C}\left(\Lambda_{2}\right.$, mixed) be the collection of chords on $\Lambda_{2}$ and mixed words of chords, so that the mixed words start and end on $\Lambda_{2}$. We say that $f$ is asymptotic to the chords $c_{1}, \ldots, c_{k}$ in $\mathcal{C}\left(\Lambda_{2}\right.$, mixed) of lengths $T_{1-}, \ldots, T_{k-}$ at the boundary punctures $z_{1-}, \ldots, z_{k-}$ at $-\infty$ if:

(1) $f$ maps pointed neighborhoods $U_{1-}, \ldots, U_{k-}$ of $z_{1-}, \ldots, z_{k-}$ into $(-\infty, 0] \times$ $Y_{0}$, so that $f(z)=(a(z), u(z))$ for all $z \in U_{1-}, \ldots, U_{k-}$.

(2) $\lim _{z \rightarrow z_{i-}} a(z)=-\infty$.

(3) In holomorphic polar coordinates $(\rho, \theta), \theta \in[0, \pi]$, centered at $z_{1-}, \ldots, z_{k-}$ (where $\theta \in\{0, \pi\}$ along $\partial \Sigma$ ), $\lim _{\rho \rightarrow 0} u(\rho, \theta)=c_{i-}\left(\frac{T_{i-}}{\pi} \theta\right)$ for $i=1, \ldots, k$.

We denote the space of holomorphic discs with asymptotic properties described above by $\mathcal{M}\left(\tilde{c} ; c_{1}, \ldots, c_{k}\right)$. Note that the additive group $\mathbb{R}$ acts freely on $\mathcal{M}\left(\widetilde{c} ; c_{1}, \ldots, c_{k}\right)$ by translations in the $t$ direction, ie $t \cdot f(z)=t \cdot(a(z), u(z)):=(t+a(z), u(z))$ in the symplectization $\mathbb{R} \times Y_{0}$. After dividing out the vertical translation we denote the moduli space by $\widehat{\mathcal{M}}\left(\widetilde{c} ; c_{1}, \ldots, c_{k}\right)=\mathcal{M}\left(\tilde{c} ; c_{1}, \ldots, c_{k}\right) / \mathbb{R}$. 
Now consider the map $\Phi: \operatorname{LHA}\left(\widetilde{\Lambda}_{2}\right) \rightarrow \operatorname{LHA}\left(\Lambda_{1} \cup \Lambda_{2}\right)$ defined by

$$
\Phi(\widetilde{c})=\sum_{\substack{\left|c_{1}\right|+\cdots+\left|c_{k}\right|=|\widetilde{c}| \\ c_{1} \cdots c_{k} \in \mathcal{C}\left(\Lambda_{1} \cup \Lambda_{2}\right)}} \# \widehat{\mathcal{M}}\left(\tilde{c} ; c_{1}, \ldots, c_{k}\right) \cdot c_{1} \cdots c_{k} .
$$

Here the summation is over the grading of $\tilde{c}$ when it is equal to the sum of the gradings of $c_{1}, \ldots, c_{k}$. For a word $\tilde{w}=\widetilde{c}_{1} \cdots \widetilde{c}_{l} \in \operatorname{LHA}\left(\tilde{\Lambda}_{2}\right)$ we define

$$
\Phi(\widetilde{w}):=\Phi\left(\tilde{c}_{1}\right) \cdots \Phi\left(\tilde{c}_{l}\right)
$$

which shows that $\Phi$ is an algebra map on $\operatorname{LHA}\left(\tilde{\Lambda}_{2}\right)$. Recall that $\operatorname{LHA}\left(\tilde{\Lambda}_{2}\right)$ consists of composable words on $\tilde{\Lambda}_{2}$, and $\operatorname{LHO}\left(\tilde{\Lambda}_{2}\right)$ consists of the composable words $\tilde{c}_{1} \cdots \tilde{c}_{m}$ in which the end point of $\tilde{c}_{m}$ lies on the same Legendrian sphere as the origin of $\widetilde{c}_{1}$. Here $\tilde{\Lambda}_{2}$ is the only Legendrian component, so $\operatorname{LHA}\left(\tilde{\Lambda}_{2}\right)=\operatorname{LHO}\left(\widetilde{\Lambda}_{2}\right)$.

As the holomorphic discs in $\widehat{\mathcal{M}}\left(\tilde{c} ; c_{1}, \ldots, c_{k}\right)$ satisfy the boundary condition $L$, the target of the map $\Phi$ is the subalgebra $\operatorname{LHA}\left(\Lambda_{2}\right.$, mixed) that consists of composable words which start and end only on $\Lambda_{2}$. So the target is $\operatorname{LHA}\left(\Lambda_{2}\right.$, mixed $)=\operatorname{LHO}\left(\Lambda_{2}\right.$, mixed $)$ with differential $d_{\left(\Lambda_{2}, \text { mixed) }\right.}$ which is the restriction of the differential $d_{\mathrm{LHA}}\left(\Lambda_{1} \cup \Lambda_{2}\right)$ to $\operatorname{LHO}\left(\Lambda_{2}\right.$, mixed). Then using the graded cyclic permutation, $\Phi$ induces the map $\Phi: \operatorname{LH}^{\text {cyc }}\left(\widetilde{\Lambda}_{2}\right) \rightarrow \operatorname{LH}^{\text {cyc }}\left(\Lambda_{2}\right.$, mixed $)$.

Proposition 3 The map $\Phi$ is a chain map, ie $\Phi \circ d_{\tilde{\Lambda}_{2}}=d_{\left(\Lambda_{2}, \text { mixed }\right)} \circ \Phi$.

Proof Consider the 1-dimensional components of the moduli space $\widehat{\mathcal{M}}\left(\widetilde{c} ; c_{1}, \ldots, c_{k}\right)$ with $|\widetilde{c}|-\left(\left|c_{1}\right|+\cdots+\left|c_{k}\right|\right)=1$ interpolating the broken trajectories. We consider one level in the symplectic cobordism and one level in the symplectization of $Y_{0}$ or $Y_{1}$. Its boundary has the form

$$
\begin{aligned}
& \bigcup \mathcal{M}^{Y_{1}}(\tilde{c} ; \widetilde{e}) / \mathbb{R} \times \widehat{\mathcal{M}}\left(\widetilde{e} ; c_{1}, \ldots, c_{k}\right) \\
& |\widetilde{c}|-1=|\widetilde{e}|= \\
& \begin{aligned}
\cup & \widehat{\mathcal{M}}\left(\tilde{c} ; c_{1}, \ldots, c_{j-1}, e, c_{j+l+1}, \ldots, c_{k}\right) \\
\begin{array}{c}
|\widetilde{c}|=\left|c_{1}\right|+\cdots+\left|c_{j-1}\right|+|e|+ \\
\left|c_{j}+l+1\right|+\cdots+\left|c_{k}\right| \\
|e|-1=\left|c_{j}\right|+\cdots+\left|c_{j+l}\right|
\end{array} & \times \mathcal{M}^{Y_{0}}\left(e ; c_{j}, \ldots, c_{j+l}\right) / \mathbb{R} .
\end{aligned}
\end{aligned}
$$

In the first type of the boundary components, $\mathcal{M}^{Y_{1}}(\widetilde{c} ; \widetilde{e}) / \mathbb{R}$ denotes the moduli space of holomorphic discs in the symplectization of $Y_{1}$ where $\tilde{e} \in \mathcal{C}\left(\tilde{\Lambda}_{2}\right)$.

In the second type of the boundary components, $\mathcal{M}^{Y_{0}}\left(e ; c_{j}, \ldots, c_{j+l}\right) / \mathbb{R}$ denotes the moduli space of holomorphic discs in the symplectization of $Y_{0}$, where $e \in$ $\mathcal{C}\left(\Lambda_{2}\right.$, mixed) and $1 \leq j \leq k, 0 \leq l \leq k-1$. Moreover, if $e$ represents a chord of the type $e^{2}, e^{21}$ or $e^{12}$, then $c_{j}, \ldots, c_{j+l}$ corresponds to an arrangement of the chords as $c_{1}^{2}, \ldots, c_{p}^{2}, c^{21}, c_{1}^{1}, \ldots, c_{r}^{1}, c^{12}, c_{1}^{2}, \ldots, c_{q}^{2}$ (for $0 \leq p, q \leq k$ and $0 \leq r \leq k-2$ ) 
or as $c_{1}^{2}, \ldots, c_{p}^{2}, c^{21}, c_{1}^{1}, \ldots, c_{r}^{1}$ or $c_{1}^{1}, \ldots, c_{r}^{1}, c^{12}, c_{1}^{2}, \ldots, c_{q}^{2}$ (for $0 \leq p, q \leq k$ and $0 \leq r \leq k-1)$ respectively.

For the special case $\widehat{\mathcal{M}}(\widetilde{c} ; \varnothing)$ (with $|\widetilde{c}|=1$ ), ie when there is no negative puncture, the boundary has the form

$$
\bigcup_{\substack{|\tilde{c}|-1=0 \\|\tilde{e}|=0}} \mathcal{M}^{Y_{1}}(\tilde{c} ; \tilde{e}) / \mathbb{R} \times \widehat{\mathcal{M}}(\tilde{e} ; \varnothing) \cup \underset{|\widetilde{c}|=|e|=1}{\bigcup} \widehat{\mathcal{M}}(\tilde{c} ; e) \times \mathcal{M}^{Y_{0}}(e ; \varnothing) / \mathbb{R} .
$$

In this case the count of the elements of the boundary components gives constant terms, however in cyclic complexes the constant terms are zero.

The compactness theorem (see Bourgeois, Eliashberg, Hofer, Wysocki and Zehnder [5]) ensures that the boundary has the form above with all moduli spaces replaced by their compactifications. In our situation the boundary is 0 -dimensional and the above moduli spaces are already compact. The count of the elements of the first type corresponds to $\Phi \circ d \tilde{\Lambda}_{2}$ whereas the count of the elements of the second type corresponds to $d_{\left(\Lambda_{2}, \text { mixed }\right)} \circ \Phi$. This shows that $\Phi \circ d \widetilde{\Lambda}_{2} \pm d_{\left(\Lambda_{2}, \text { mixed }\right)} \circ \Phi=0$. Then the relation $\Phi \circ d \tilde{\Lambda}_{2}=d_{\left(\Lambda_{2}, \text { mixed }\right)} \circ \Phi$ is obtained from the fact that orientations are coherent with the gluing operation; for more details see Eliashberg, Givental and Hofer [12].

Therefore the induced map $\Phi: \mathrm{LH}^{\mathrm{cyc}}\left(\widetilde{\Lambda}_{2}\right) \rightarrow \mathrm{LH}^{\mathrm{cyc}}\left(\Lambda_{2}\right.$, mixed) is also a chain map. This chain map induces the map $\bar{\Phi}: L \mathbb{H}^{\text {cyc }}\left(\tilde{\Lambda}_{2}\right) \rightarrow L \mathbb{H}^{\text {cyc }}\left(\Lambda_{2}\right.$, mixed $)$ in the level of homology.

Proposition 4 The map $\Phi$ is an isomorphism.

Sketch of the proof Recall that the symplectic cobordism $W_{1}$ is a Liouville manifold, that is $L_{X} \omega=\omega$ or $d\left(i_{X} \omega\right)=\omega$, which means $\omega$ is an exact form with the primitive $\lambda=i_{X} \omega$ and $\left.\lambda\right|_{Y_{0}}=\lambda_{0}$ and $\left.\lambda\right|_{Y_{1}}=\lambda_{1}$. Regarding the solution $f$ of the equation $d f \circ j=J \circ d f$, equivalently $\partial_{x} f+J(f) \partial_{y} f=0, z=x+i y \in \mathbb{C}$, by compatibility of the almost complex structure $J$ with the symplectic form, we have $d \lambda\left(\partial_{x} f, \partial_{y} f\right)=$ $d \lambda\left(\partial_{x} f, J \partial_{x} f\right) \geq 0$. Therefore $\int_{\Sigma} f^{*} d \lambda \geq 0$. This means that $\Phi$ decreases the contact action $\tilde{c} \mapsto \int_{[0,1]} \tilde{c}^{*} \lambda$. We arrange the generators of the complex $\operatorname{LH}^{\mathrm{cyc}}\left(\widetilde{\Lambda}_{2}\right)$ in decreasing order according to their action. Then the matrix $\Phi$ in this basis is upper triangular. We claim that the entries on the diagonal are all equal \pm 1 , which implies that $\Phi$ is an isomorphism.

If the word $\tilde{w}$ contains only the letters in correspondence to the chords of $\Lambda_{2}$ which are unaffected by the surgery then the diagonal value is equal to \pm 1 . Because the diagonal value corresponds to the trivial holomorphic disc in which the cyclic words in the top and bottom of the cobordism $W_{1}$ have the same length. 
Now consider the chords which are created by the surgery. We consider the simplest case, ie when $\tilde{c}$ in $Y_{1}$ is the created chord in correspondence to $c^{21} c^{12}$ in $Y_{0}$. The created chord $\tilde{c}$ is the Reeb chord which contains the Reeb trajectories that are in a $\delta$-neighborhood of $c^{21}$ and $c^{12}$ in $\Lambda_{1} \sqcup \Lambda_{2}$, and the Reeb trajectory which is passing through the handle. When the size of the attaching handle goes to zero, the length of this Reeb trajectory will be very short as $\varepsilon>0$. So we have $\int_{\Sigma} f^{*} d \lambda=$ $\mathcal{A}(\widetilde{c})-\left(\mathcal{A}\left(c^{21}\right)+\mathcal{A}\left(c^{12}\right)\right)=\varepsilon>0$. Hence $\int_{\Sigma} f^{*} d \lambda \sim 0$ implies that $f$ is an almost vertical holomorphic disc.

We would like to show that such a holomorphic disc, denoted by $L$, is unique. For this aim we show that the holomorphic strip $D$ asymptotic to $c^{21}$ in the bottom of cobordism and asymptotic to $c^{21}$ and $\frac{\gamma}{2}$ in the top of the cobordism is unique. Here $\frac{\gamma}{2}$ is the half of the Reeb chord $\gamma$ of the cocore sphere of the attaching handle.

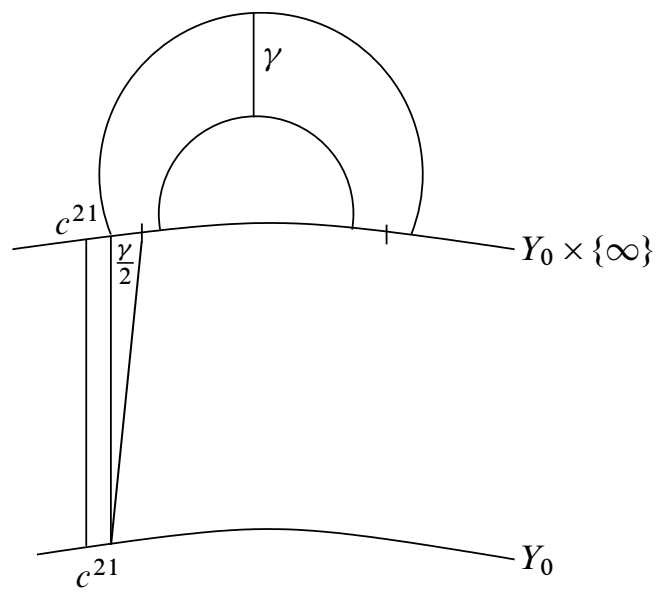

Figure 5: Holomorphic strip in surgery cobordism

In fact we monitor the Reeb trajectory inside the attaching handle by the Reeb chord of the cocore sphere which has a very small length as $\varepsilon>0$. In [4] it is explained that whenever we perform surgery by attaching handle, we obtain Reeb chords for the Legendrian cocore sphere which are in correspondence to the Reeb chords of the Legendrian sphere along which we performed surgery.

As the difference of actions is $\varepsilon / 2$, in fact we can consider many holomorphic strips (in a flat complex line) with the above description.

We note that any other disc with the given properties lies in a small neighborhood of $D$. Since if the disc is not contained in a small neighborhood of $D$ then its area is bigger than $\varepsilon / 2$. 

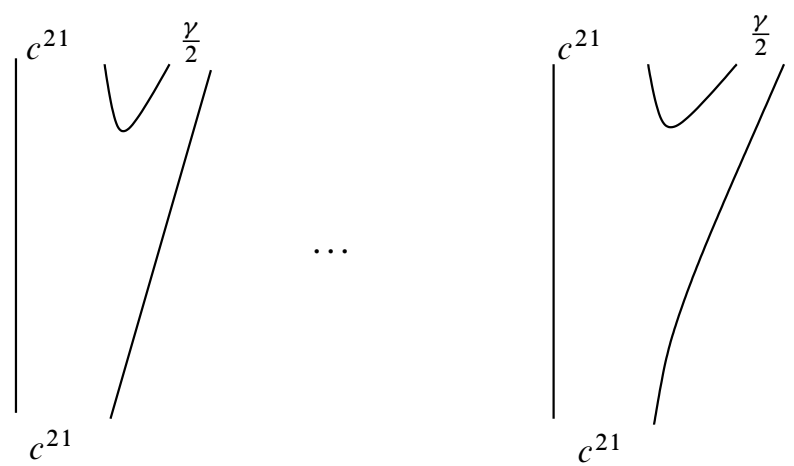

Figure 6: Possible holomorphic strips

According to [4], in a suitable model for the handle the disc $D$ lies in a flat complex line, and the linearization of the $\bar{\partial}$-operator at disc $D$ is surjective and the parameterized moduli space is a 1-manifold (for the definition of the operator $\bar{\partial}$ and the relevant analysis setup see [16]). Therefore by [4] the holomorphic strip $D$ is unique in its small neighborhood.

Similarly, the holomorphic strip $D^{\prime}$, asymptotic to $c^{12}$ in the bottom of the cobordism and asymptotic to $\frac{\gamma}{2}$ and $c^{12}$ in top of the cobordism, is unique.

In fact to create disc $D^{\prime}$ using the standard model we must move the Reeb chords of the previously constructed disc $D$. Because of action there can be no splittings so the moduli space undergoes cobordism.

Next we glue two holomorphic strips $D$ and $D^{\prime}$, and because of action there is only one splitting which gives the desired disc $L$.

Finally using above inductively, because of action argument in each step there is no splitting and we obtain the uniqueness of holomorphic discs in correspondence to the cyclic words $\widetilde{c}_{k} \sim c^{21} c_{1}^{1} \cdots c_{k}^{1} c^{12}$.

All above argument leads to the following theorem.

Theorem 2 We have that $L \mathbb{H}^{\text {cyc }}\left(\widetilde{\Lambda}_{2}\right) \cong L \mathbb{H}^{\text {cyc }}\left(\Lambda_{2}\right.$, mixed) as cyclic Legendrian homologies. Therefore the long exact sequence in (1) turns to the following long exact sequence:

$$
\cdots \rightarrow L \mathbb{H}_{k}^{\mathrm{cyc}}\left(\tilde{\Lambda}_{2}\right) \rightarrow L \mathbb{H}_{k}^{\mathrm{cyc}}(\Lambda) \rightarrow L \mathbb{H}_{k}^{\mathrm{cyc}}\left(\Lambda_{1}\right) \rightarrow L \mathbb{H}_{k-1}^{\mathrm{cyc}}\left(\tilde{\Lambda}_{2}\right) \rightarrow \cdots
$$

Remark 4 When the Legendrian link has more components, we can repeat all above construction. For instance, consider a Legendrian link with three components, ie 
$\Lambda=\Lambda_{1} \sqcup \Lambda_{2} \sqcup \Lambda_{3}$. After performing Legendrian surgery along $\Lambda_{1}$ in the contact manifold $Y_{0}$ we perform a Legendrian surgery along $\widetilde{\Lambda}_{2}$ in the contact manifold $Y_{1}$. The chords in $\mathrm{LH}^{\text {cyc }}\left(\widetilde{\Lambda}_{3}\right)$ have two types: the chords of $\Lambda_{3}$ unaffected by the surgery and the created chords $\widetilde{c}_{3}$ in correspondence to the words $c^{31} c_{1}^{1} \cdots c_{k}^{1} c^{13}$ and $c^{32} \widetilde{c}_{1}^{2} \cdots \tilde{c}_{k}^{2} c^{23}$.

\section{Examples}

Consider $\Lambda_{1} \sqcup \Lambda_{2}$ as a Legendrian link of the Legendrian spheres in the contact manifold $Y_{0}$. After performing Legendrian surgery along $\Lambda_{1}$ and following the instruction given in the previous section, here we would like to describe the cyclic Legendrian homology of $\tilde{\Lambda}_{2}$. Recall that the module $\operatorname{LH}^{\text {cyc }}\left(\tilde{\Lambda}_{2}\right)$ is generated by the cyclic words in chords of $\tilde{\Lambda}_{2}$ in which the chords have two types: the chords of $\Lambda_{2}$ unaffected by the surgery and the chords created by the surgery which are in one-to-one correspondence with the words of the type $c_{1}^{21} c_{1}^{1} \cdots c_{k}^{1} c_{1}^{12}$.

\subsection{Cyclic Legendrian homology of the Hopf link in $S^{3}$}

Let $\Lambda=\Lambda_{1} \sqcup \Lambda_{2} \subset S^{3}$ be the Legendrian link with two components which are both the simple Legendrian unknots. We can concentrate only on the Darboux ball containing $\Lambda$, so that our computations will be the same as if $\Lambda$ is a Legendrian link in $\mathbb{R}^{3}$ with the standard contact structure. We illustrate this link in front projection in the following diagram.

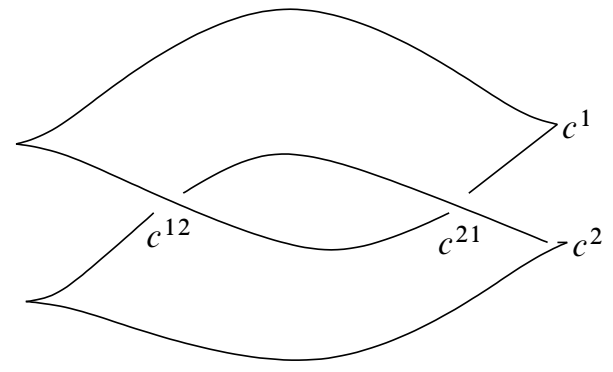

Figure 7: $\Lambda$ in front projection

The Reeb chords are in correspondence to $c^{1}, c^{2}, c^{21}, c^{12}$ which denote respectively a Reeb chord on $\Lambda_{1}$, a Reeb chord on $\Lambda_{2}$, a Reeb chord from $\Lambda_{2}$ to $\Lambda_{1}$, and a Reeb chord from $\Lambda_{1}$ to $\Lambda_{2}$. In fact by Ng's description (see [17]) of the Chekanov DGA (see [6]) in the front projection, the generators are double points and right cusp points in the front projection. Using the index formula in front projection given by Ekholm, 
Etnyre and Sullivan in [10, Lemma 3.4] (also see Section 3.3), the gradings for these Reeb chords are

$$
\left|c^{1}\right|=\left|c^{2}\right|=1 \quad \text { and } \quad\left|c^{21} c^{12}\right|=0 .
$$

Moreover the differential is

$$
\partial c^{1}=1-1+c^{12} c^{21}, \quad \partial c^{2}=1-1+c^{21} c^{12}, \quad \partial c^{21}=\partial c^{12}=0 .
$$

Remark 5 As it is shown by Ekholm, Etnyre and Sullivan in [11], the differential $\partial$ depends on the particular spin structure on $\Lambda$. Any component of $\Lambda$ has two spin structures: the Lie group spin structure on $S^{1}$, and the nullcobordant spin structure on $S^{1}$. Here we use the nullcobordant spin structure on any component of $\Lambda$.

In $\tilde{\Lambda}_{2}$ the chords are $c^{2}$ and $\tilde{c}_{l}:=c^{21}\left(c^{1}\right)^{l} c^{12}, l \geq 0$, where $\left|c^{2}\right|=1$ and $\left|\widetilde{c}_{l}\right|=l$. Then we have

$$
\partial c^{2}=c^{21} c^{12}=\widetilde{c}_{0}
$$

and using the Leibniz rule we have

$$
\begin{aligned}
\partial \widetilde{c}_{l}=\partial\left(c^{21}\left(c^{1}\right)^{l} c^{12}\right) & =\sum_{j=1}^{l}(-1)^{j-1} c^{21}\left(c^{1}\right)^{j-1} c^{12} c^{21}\left(c^{1}\right)^{l-j} c^{12} \\
& =\sum_{j=1}^{l}(-1)^{j} \widetilde{c}_{j-1} \widetilde{c}_{l-j} .
\end{aligned}
$$

Hence we observe that $\partial \widetilde{c}_{1}=-\widetilde{c}_{0} \widetilde{c}_{0}$ and $\partial \widetilde{c}_{2}=-\widetilde{c}_{0} \widetilde{c}_{1}+\widetilde{c}_{1} \widetilde{c}_{0} \stackrel{\text { cyc }}{=} 0$. More generally, $\partial \widetilde{c}_{2 l} \stackrel{\text { cyc }}{=} 0$ for each $l \geq 1$. The cyclic complex is as follows:

$\operatorname{LH}_{-1}^{\mathrm{cyc}}\left(\widetilde{\Lambda}_{2}\right)=0$

$\operatorname{LH}_{0}^{\mathrm{cyc}}\left(\widetilde{\Lambda}_{2}\right)=\left\langle\left(\widetilde{c}_{0}^{k}\right) \mid k \geq 1\right\rangle$

$\mathrm{LH}_{1}^{\mathrm{cyc}}\left(\widetilde{\Lambda}_{2}\right)=\left\langle\left(\widetilde{c}_{1} \widetilde{c}_{0}^{k}\right),\left(c^{2} \widetilde{c}_{0}^{k}\right) \mid k \geq 0\right\rangle$

$\mathrm{LH}_{2}^{\mathrm{cyc}}\left(\widetilde{\Lambda}_{2}\right)=\left\langle\left(\widetilde{c}^{2} \widetilde{c}_{0}^{p}\right),\left(c^{2} \widetilde{c}_{0}^{m} c^{2} \widetilde{c}_{0}^{n}\right),\left(c^{2} \widetilde{c}_{0}^{p} \widetilde{c}_{1} \widetilde{c}_{0}^{q}\right),\left(\widetilde{c}_{1} \widetilde{c}_{0}^{m} \widetilde{c}_{1} \widetilde{c}_{0}^{n}\right) \mid p, q, m, n \geq 0, m \neq n\right\rangle$

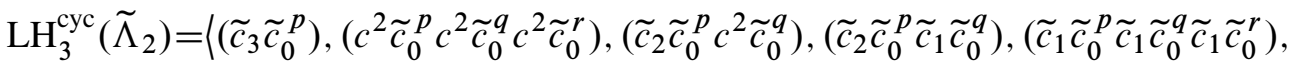

$$
\left(c^{2} \widetilde{c}_{0}^{p} c^{2} \widetilde{c}_{0}^{q} \widetilde{c}_{1} \tilde{c}_{0}^{r}\right),\left(\tilde{c}_{1} \tilde{c}_{0}^{p} \widetilde{c}_{1} \tilde{c}_{0}^{q} c^{2} \widetilde{c}_{0}^{r}\right)|p, q, r \geq 0\rangle
$$


The cyclic contact homology in degree zero is equal to

$$
L \mathbb{H}_{0}^{\mathrm{cyc}}\left(\tilde{\Lambda}_{2}\right)=\frac{\operatorname{ker} \partial_{0}^{\mathrm{cyc}}}{\operatorname{im} \partial_{1}^{\mathrm{cyc}}}=\frac{\operatorname{LH}_{0}^{\mathrm{cyc}}\left(\tilde{\Lambda}_{2}\right)}{\operatorname{LH}_{0}^{\mathrm{cyc}}\left(\tilde{\Lambda}_{2}\right)}=0 .
$$

Since $\partial_{0}^{\text {cyc }}\left(\tilde{c}_{0}\right)=0$ and $\operatorname{im} \partial_{1}^{\text {cyc }}=\operatorname{LH}_{0}^{\text {cyc }}\left(\widetilde{\Lambda}_{2}\right)$. We know this because by the Leibniz rule we have

$$
\partial_{1}^{\mathrm{cyc}}\left(\tilde{c}_{1} \tilde{c}_{0}^{k}\right)=-\tilde{c}_{0}^{k+2}, \quad \partial_{1}^{\mathrm{cyc}}\left(c^{2} \tilde{c}_{0}^{k}\right)=\tilde{c}_{0}^{k+1}, \quad k \geq 0 .
$$

From the above computation, we immediately conclude that

$$
\operatorname{ker} \partial_{1}^{\mathrm{cyc}}=\left\langle\left(\tilde{c}_{1} \tilde{c}_{0}^{k}+c^{2} \tilde{c}_{0}^{k+1}\right) \mid k \geq 0\right\rangle .
$$

Moreover, im $\partial_{2}^{\text {cyc }}$ is obtained by the following:

$$
\begin{aligned}
\partial_{2}^{\mathrm{cyc}}\left(\widetilde{c}_{2} \tilde{c}_{0}^{p}\right) & =0, & & \partial_{2}^{\mathrm{cyc}}\left(c^{2} \widetilde{c}_{0}^{m} c^{2} \widetilde{c}_{0}^{n}\right)=0, \\
\partial_{2}^{\mathrm{cyc}}\left(c^{2} \widetilde{c}_{0}^{p} \widetilde{c}_{1} \tilde{c}_{0}^{q}\right) & =\tilde{c}_{1} \tilde{c}_{0}^{p+q+1}+c^{2} \widetilde{c}_{0}^{p+q+2}, & & \partial_{2}^{\mathrm{cyc}}\left(\widetilde{c}_{1} \tilde{c}_{0}^{m} \widetilde{c}_{1} \widetilde{c}^{n}\right)=0 .
\end{aligned}
$$

In other words, we have

$$
\operatorname{im} \partial_{2}^{\mathrm{cyc}}=\left\langle\left(\widetilde{c}_{1} \tilde{c}_{0}^{k}+c^{2} \tilde{c}_{0}^{k+1}\right) \mid k \geq 1\right\rangle .
$$

Therefore the cyclic Legendrian homology in degree one is equal to

$$
L \mathbb{H}_{1}^{\mathrm{cyc}}\left(\widetilde{\Lambda}_{2}\right)=\frac{\operatorname{ker} \partial_{1}^{\mathrm{cyc}}}{\operatorname{im} \partial_{2}^{\mathrm{cyc}}}=\left\langle\left(\widetilde{c}_{1}+c^{2} \widetilde{c}_{0}\right)\right\rangle .
$$

From above we also conclude that

$$
\operatorname{ker} \partial_{2}^{\mathrm{cyc}}=\left\langle\left(\widetilde{c}_{2} \tilde{c}_{0}^{p}\right),\left(c^{2} \tilde{c}_{0}^{m} c^{2} \widetilde{c}_{0}^{n}\right),\left(\tilde{c}_{1} \tilde{c}_{0}^{m} \tilde{c}_{1} \tilde{c}_{0}^{n}\right) \mid p, m, n \geq 0, m \neq n\right\rangle .
$$

On the other hand the image $\operatorname{im} \partial_{3}^{\text {cyc }}$ is obtained by the following:

$$
\begin{aligned}
& \partial_{3}^{\mathrm{cyc}}\left(\widetilde{c}_{3} \tilde{c}_{0}^{p}\right)=-2 \widetilde{c}_{2} \widetilde{c}_{0}^{p+1}+\widetilde{c}_{1}^{2} \widetilde{c}_{0}^{p}, \\
& \partial_{3}^{\mathrm{cyc}}\left(c^{2} \widetilde{c}_{0}^{p} c^{2} \widetilde{c}_{0}^{q} c^{2} \widetilde{c}_{0}^{r}\right)=c^{2} \widetilde{c}_{0}^{q} c^{2} \widetilde{c}_{0}^{p+r+1}+c^{2} \widetilde{c}_{0}^{r} c^{2} \widetilde{c}_{0}^{p+q+1}+c^{2} \widetilde{c}_{0}^{p} c^{2} \widetilde{c}_{0}^{q+r+1} \text {, } \\
& \partial_{3}^{\mathrm{cyc}}\left(\widetilde{c}_{2} \widetilde{c}_{0}^{p} c^{2} \widetilde{c}_{0}^{q}\right)=-\widetilde{c}_{1} \tilde{c}_{0}^{p} c^{2} \widetilde{c}_{0}^{q+1}+\widetilde{c}_{1} \widetilde{c}_{0}^{p+1} c^{2} \widetilde{c}_{0}^{q}+\widetilde{c}_{2} \widetilde{c}_{0}^{p+q+1}, \\
& \partial_{3}^{\mathrm{cyc}}\left(\widetilde{c}_{2} \widetilde{c}_{0}^{p} \widetilde{c}_{1} \tilde{c}_{0}^{q}\right)=-\widetilde{c}_{1} \tilde{c}_{0}^{p} \widetilde{c}_{1} \tilde{c}_{0}^{q+1}+\tilde{c}_{1} \tilde{c}_{0}^{p+1} \widetilde{c}_{1} \widetilde{c}_{0}^{q}-\tilde{c}_{2} \tilde{c}_{0}^{p+q+2}, \\
& \partial_{3}^{\mathrm{cyc}}\left(\widetilde{c}_{1} \widetilde{c}_{0}^{p} \widetilde{c}_{1} \widetilde{c}_{0}^{q} \widetilde{c}_{1} \widetilde{c}_{0}^{r}\right)=-\widetilde{c}_{1} \widetilde{c}_{0}^{q} \widetilde{c}_{1} \widetilde{c}_{0}^{p+r+2}-\widetilde{c}_{1} \widetilde{c}_{0}^{r} \widetilde{c}_{1} \widetilde{c}_{0}^{p+q+2}-\widetilde{c}_{1} \widetilde{c}_{0}^{p} \widetilde{c}_{1} \widetilde{c}_{0}^{q+r+2}, \\
& \partial_{3}^{\mathrm{cyc}}\left(c^{2} \widetilde{c}_{0}^{p} c^{2} \widetilde{c}_{0}^{q} \widetilde{c}_{1} \widetilde{c}_{0}^{r}\right)=c^{2} \widetilde{c}_{0}^{q} \widetilde{c}_{1} \widetilde{c}_{0}^{p+r+1}-c^{2} \widetilde{c}_{0}^{p+q+1} \widetilde{c}_{1} \widetilde{c}_{0}^{r}-c^{2} \widetilde{c}_{0}^{p} c^{2} \widetilde{c}_{0}^{q+r+2} \text {, } \\
& \partial_{3}^{\mathrm{cyc}}\left(\widetilde{c}_{1} \tilde{c}_{0}^{p} \widetilde{c}_{1} \widetilde{c}_{0}^{q} c^{2} \widetilde{c}_{0}^{r}\right)=-\widetilde{c}_{1} \widetilde{c}_{0}^{q} c^{2} \widetilde{c}_{0}^{p+r+2}+\widetilde{c}_{1} \widetilde{c}_{0}^{p+q+2} c^{2} \widetilde{c}_{0}^{r}+\widetilde{c}_{1} \widetilde{c}_{0}^{p} \widetilde{c}_{1} \widetilde{c}_{0}^{q+r+1} \text {. }
\end{aligned}
$$


Therefore the cyclic Legendrian homology in degree two is equal to

$$
L \mathbb{H}_{2}^{\mathrm{cyc}}\left(\tilde{\Lambda}_{2}\right)=\frac{\operatorname{ker} \partial_{2}^{\mathrm{cyc}}}{\operatorname{im} \partial_{3}^{\mathrm{cyc}}}=\left\langle\left(\tilde{c}_{2}\right)\right\rangle .
$$

In general we see that $\left(\tilde{c}_{2 k}\right)$ (when $k \neq 0$ ) is the generator in even degrees, and $\left(\widetilde{c}_{1}+c^{2} \widetilde{c}_{0}\right)^{2 k+1}$ is the generator in odd degrees. Therefore the cyclic Legendrian homology of $\tilde{\Lambda}_{2}$ is as follows:

$$
L \mathbb{H}_{k}^{\mathrm{cyc}}\left(\tilde{\Lambda}_{2}\right)= \begin{cases}0 & k=0 \\ \left\langle\left(\widetilde{c}_{k}\right)\right\rangle & k>0 \text { even } \\ \left\langle\left(\widetilde{c}_{1}+c^{2} \widetilde{c}_{0}\right)^{k}\right\rangle & k \geq 1 \text { odd }\end{cases}
$$

Now, using $L \mathbb{H}_{k}^{\mathrm{cyc}}\left(\widetilde{\Lambda}_{2}\right)$ in the exact sequence found in Theorem 2, we can compute $L \mathbb{H}^{\mathrm{cyc}}\left(\Lambda_{1} \sqcup \Lambda_{2}\right)$.

First note that $L \mathbb{H}_{2 k}^{\mathrm{cyc}}\left(\Lambda_{1}\right)$ is zero because there is no generator in even degrees, since $\left(c^{1}\right)^{2 k}$ is not a good word. In odd degrees we have

$$
L \mathbb{H}_{2 k+1}^{\mathrm{cyc}}\left(\Lambda_{1}\right)=\left\langle\left(\left(c^{1}\right)^{2 k+1}\right)\right\rangle,
$$

because $\partial\left(c^{1}\right)=c^{12} c^{21} \stackrel{\text { cyc }}{=} c^{21} c^{12} \in \operatorname{LH}_{0}^{\text {cyc }}\left(\widetilde{\Lambda}_{2}\right)$, where $\operatorname{LH}_{0}^{\text {cyc }}\left(\widetilde{\Lambda}_{2}\right)$ is the zero of the quotient complex $\operatorname{LH}_{0}^{\text {cyc }}\left(\Lambda_{1}\right)$. Also one can see that

$$
\partial\left(\left(c^{1}\right)^{2 k+1}\right) \stackrel{\text { cyc }}{=}(2 k+1)\left(\widetilde{c}_{2 k}\right) \in \operatorname{LH}_{2 k}^{\mathrm{cyc}}\left(\widetilde{\Lambda}_{2}\right)
$$

which is the zero of the quotient complex $\operatorname{LH}_{2 k}^{\mathrm{cyc}}\left(\Lambda_{1}\right)$.

Consider the exact sequence

$$
\rightarrow L \mathbb{H}_{2 k+1}^{\mathrm{cyc}}\left(\tilde{\Lambda}_{2}\right) \rightarrow L \mathbb{H}_{2 k+1}^{\mathrm{cyc}}\left(\Lambda_{1} \sqcup \Lambda_{2}\right) \rightarrow L \mathbb{H}_{2 k+1}^{\mathrm{cyc}}\left(\Lambda_{1}\right) \stackrel{d_{2 k+1}}{\longrightarrow} L \mathbb{H}_{2 k}^{\mathrm{cyc}}\left(\tilde{\Lambda}_{2}\right) \rightarrow \text {. }
$$

For the element $\left[\left(c^{1}\right)^{2 k+1}+\operatorname{LH}_{2 k+1}^{\mathrm{cyc}}\left(\widetilde{\Lambda}_{2}\right)\right]$ of the homology $L \mathbb{H}_{2 k+1}^{\mathrm{cyc}}\left(\Lambda_{1}\right)$ we have

$$
\begin{aligned}
d_{2 k+1}\left(\left[\left(c^{1}\right)^{2 k+1}+\operatorname{LH}_{2 k+1}^{\mathrm{cyc}}\left(\widetilde{\Lambda}_{2}\right)\right]\right) & =i_{2 k}^{-1} \partial p^{-1}\left(\left[\left(c^{1}\right)^{2 k+1}+\operatorname{LH}_{2 k+1}^{\mathrm{cyc}}\left(\widetilde{\Lambda}_{2}\right]\right)\right. \\
& =i_{2 k}^{-1} \partial\left(\left[\left(c^{1}\right)^{2 k+1}\right]\right)=i_{2 k}^{-1}\left(\left[\tilde{c}_{2 k}\right]\right)=i_{2 k}^{-1}(0)=0,
\end{aligned}
$$

where $i$ and $p$ are respectively the injective and surjective maps in the short exact sequence of the complexes. Here we have used the fact that the homology class of $\tilde{c}_{2 k}$ in $L \mathbb{H}_{2 k}^{\text {cyc }}\left(\Lambda_{1} \sqcup \Lambda_{2}\right)$ is zero, because $\partial\left(c^{1}\right)^{2 k+1}=\tilde{c}_{2 k}$. Since $d_{2 k+1}$ is a zero map, the exactness of the above sequence and (2) imply that $L \mathbb{H}_{2 k+1}^{\mathrm{cyc}}\left(\Lambda_{1} \sqcup \Lambda_{2}\right)=$ $\left\langle\left(\widetilde{c}_{1}+c^{2} \widetilde{c}_{0}\right)^{2 k+1},\left(\left(c^{1}\right)^{2 k+1}\right)\right\rangle$. 
Let $x$ be a cycle in $\operatorname{LH}_{2 k}^{\text {cyc }}\left(\Lambda_{1} \sqcup \Lambda_{2}\right)$, ie $\partial(x)=0$. Then $x+\operatorname{LH}_{2 k}^{\text {cyc }}\left(\widetilde{\Lambda}_{2}\right)$ is a cycle in $\operatorname{LH}_{2 k}^{\mathrm{cyc}}\left(\Lambda_{1}\right)$. The equation $L \mathbb{H}_{2 k}^{\mathrm{cyc}}\left(\Lambda_{1}\right)=0$ means that all cycles in $\operatorname{LH}_{2 k}^{\mathrm{cyc}}\left(\Lambda_{1}\right)$ are trivial. Hence $x+\operatorname{LH}_{2 k}^{\mathrm{cyc}}\left(\tilde{\Lambda}_{2}\right)$ is a trivial cycle, ie

$$
x+\operatorname{LH}_{2 k}^{\mathrm{cyc}}\left(\tilde{\Lambda}_{2}\right)=\partial z+\operatorname{LH}_{2 k}^{\mathrm{cyc}}\left(\tilde{\Lambda}_{2}\right)
$$

for a $z \in \operatorname{LH}_{2 k+1}^{\text {cyc }}\left(\Lambda_{1} \sqcup \Lambda_{2}\right)$. So $x-\partial z \in \operatorname{LH}_{2 k}^{\mathrm{cyc}}\left(\tilde{\Lambda}_{2}\right)$. We set $x-\partial z:=y$, so $y$ is a cycle. By

$$
L \mathbb{H}_{2 k}^{\mathrm{cyc}}\left(\tilde{\Lambda}_{2}\right)=\left\langle\left(\tilde{c}_{2 k}\right)\right\rangle
$$

we see that all the cycles in $\operatorname{LH}_{2 k}^{\mathrm{cyc}}\left(\widetilde{\Lambda}_{2}\right)$ are trivial except $\tilde{c}_{2 k}=\partial\left(\left(c^{1}\right)^{2 k+1}\right)$. So $x$ is a trivial cycle, hence $L \mathbb{H}_{2 k}^{\mathrm{cyc}}\left(\Lambda_{1} \sqcup \Lambda_{2}\right)=0$.

In summary the cyclic Legendrian homology of the link $\Lambda_{1} \sqcup \Lambda_{2}$ is as follows:

$$
L \mathbb{H}_{k}^{\mathrm{cyc}}\left(\Lambda_{1} \sqcup \Lambda_{2}\right)= \begin{cases}0 & k \geq 0 \text { even, } \\ \left\langle\left(\widetilde{c}_{1}+c^{2} \widetilde{c}_{0}\right)^{k},\left(\left(c^{1}\right)^{k}\right)\right\rangle & k \geq 1 \text { odd. }\end{cases}
$$

\subsection{Examples of lens spaces}

For several lens spaces we compute their linearized contact homology using the classification theorem of tight contact structures of lens spaces (which will be stated in Theorem 3), the surgery exact sequence in Theorem 1, and the exact sequence in Theorem 2. The linearized contact homologies of the unique tight contact structures on the lens spaces $L(2,1)$ and $L(3,2)$ are already known via the Morse-Bott techniques developed by Bourgeois [1], here using our method we compute them. The interesting cases are the lens spaces admitting several tight contact structures (see Honda [15]), and correspond to those contact structures that are not $S^{1}$-invariant and transverse to the $S^{1}$-orbits [1], since their linearized contact homologies are not known. Here we compute the linearized contact homologies of $L(2 r+1,2), r \geq 2$, with its different tight contact structures.

3.2.1 Linearized contact homology of $\left(\mathbb{R} \boldsymbol{P}^{\mathbf{3}}, \boldsymbol{\xi}_{\mathrm{st}}\right)$ Let $\Lambda_{1}$ be the simple Legendrian unknot in the contact manifold $\left(S^{3}, \xi_{\text {st }}\right)$ with the following diagram in $\mathbb{R}^{3}$.

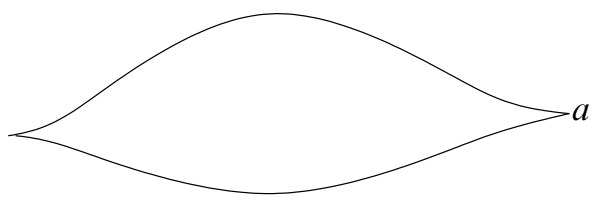

Figure 8: $\Lambda_{1}$ in front projection 
We perform the Legendrian surgery along $\Lambda_{1}$. In fact the new contact manifold obtaining by the Legendrian surgery along $\Lambda_{1}$ in $S^{3}$ is the lens space $L(2,1)=\mathbb{R} P^{3}$ with its standard contact structure. This is a result of the classification of tight contact structures given by Honda which we rephrase it in the following.

Consider the lens space $L(p, q)$ where $p>q>0$ and $(p, q)=1$. Assume $-p / q$ has the continued fraction expansion

$$
-\frac{p}{q}=r_{0}-\frac{1}{r_{1}-\frac{1}{r_{2}-\cdots \frac{1}{r_{k}}}}
$$

with all $r_{i}<-1$.

Theorem 3 (Honda [15]) There exist exactly $\left|\left(r_{0}+1\right)\left(r_{1}+1\right) \cdots\left(r_{l}+1\right)\right|$ tight contact structures on the lens space $L(p, q)$ up to isotopy, where $r_{0}, \ldots, r_{l}$ are the coefficients of the continued fraction expansion of $-p / q$. Moreover, all the tight contact structures on $L(p, q)$ can be obtained from Legendrian surgery on links in $S^{3}$.

In fact in this theorem it is shown that we have a linked chain of unknots in $S^{3}$ with framings $r_{0}, r_{1}, \ldots, r_{k}$ (in order along the chain), along which we can perform Legendrian surgery to obtain $L(p, q)$. Denoting the unknots by $\gamma_{0}, \ldots, \gamma_{k}$, to perform Legendrian surgery, $\gamma_{i}$ must have Thurston-Bennequin invariant $\operatorname{tb}\left(\gamma_{i}\right)=r_{i}+1$.

For the lens space $L(2,1)$, in the continued fractions expression of $-p / q=-2 / 1$, we have $r_{0}=-2$. So by the theorem there exists only $\left|\left(r_{0}+1\right)\right|=1$ tight contact structures on $L(2,1)$ up to isotopy which can be obtained from Legendrian surgery along the Legendrian unknot with Thurston-Bennequin number tb $=-1$ in $S^{3}$.

Now we compute the cyclic Legendrian homology for $\Lambda_{1}$ : the grading of the only generator $a$ is $|a|=1$ and its differential is $\partial a=1-1=0$.

The even powers of $a$ are bad. So there is no module in even degrees. Therefore the cyclic Legendrian homology of $\Lambda_{1}$ is

$$
L \mathbb{H}_{k}^{\mathrm{cyc}}\left(\Lambda_{1}\right)= \begin{cases}\left\langle\left(a^{k}\right)\right\rangle & k \geq 1 \text { odd } \\ 0 & k \text { even. }\end{cases}
$$

On the other hand the Linearized contact homology of $S^{3}$ is given by the following theorem.

Theorem 4 (Bourgeois, Cieliebak and Ekholm [3]) Let $\lambda$ be a dynamically convex form for the tight contact structure on $S^{3}$. Let $\left(\mathcal{C}\left(S^{3}, \lambda\right), \partial_{\epsilon}\right)$ be the linearized contact complex for $\left(S^{3}, \lambda\right)$. Then the Reeb filed $R_{\lambda}$ has exactly 2 closed orbits if and only if $\partial_{\epsilon}=0$. 
Here a contact form $\lambda$ is called dynamically convex if all closed Reeb orbits are nondegenerate and have Conley-Zehnder index at least 3 .

In fact in this theorem it is shown that there is a unique (not necessarily simple) closed Reeb orbit of every positive even degree (or positive odd Conley-Zehnder index) and no other Reeb orbits. Therefore $\partial_{\epsilon}=0$ implies that $\mathbb{H} C^{\epsilon}\left(S^{3}, \xi_{\mathrm{st}}\right)=\mathcal{C}\left(S^{3}, \lambda\right)$ has one generator in each positive even degree (or positive odd Conley-Zehnder index). In [4] the gradings are considered as Conley-Zehnder indices. So in order to apply the surgery exact sequence, we consider the gradings as Conley-Zehnder indices.

In degree one there is only the generator $(a)$ for $L \mathbb{H}^{\text {cyc }}\left(\Lambda_{1}\right)$. In the other positive odd degrees we have the generator $\gamma^{k}$ for $\mathbb{H} C^{\varepsilon}\left(S^{3}\right)$, and the generator $\left(a^{k}\right)$ for $L \mathbb{H}^{\text {cyc }}\left(\Lambda_{1}\right)$, where $k>1$ is odd. Using the surgery exact sequence of Theorem 1 , the linearized contact homology of $\mathbb{R} P^{3}$ is as follows:

$$
\mathbb{H} C_{k}^{\operatorname{lin}}\left(\mathbb{R} P^{3}\right)= \begin{cases}\langle(a)\rangle & \text { for } k=1, \\ \left\langle\gamma^{k},\left(a^{k}\right)\right\rangle & \text { for } k>1 \text { odd }, \\ 0 & \text { otherwise. }\end{cases}
$$

\subsubsection{Linearized contact homology of the Lens space $L(3,2)$ By Honda's the-} orem (Theorem 3 ) the Lens space $L(3,2)$ with the continued fractions expression $-\frac{p}{q}=-\frac{3}{2}=-2+\frac{1}{2}$ has up to isotopy $|(-2+1)(-2+1)|=1$ tight contact structure. This is obtained by Legendrian surgery along Hopf link $\Lambda$ in $S^{3}$.

After surgery along the component $\Lambda_{1}$, we obtain the new contact manifold $\mathbb{R} P^{3}$ containing $\widetilde{\Lambda}_{2}$. Using the cyclic Legendrian homology $L \mathbb{H}^{\text {cyc }}\left(\tilde{\Lambda}_{2}\right)$ in $(2)$ and the linearized contact homology of $\mathbb{R} P^{3}$ in (4) in the surgery exact sequence Theorem 1 for the second surgery along $\widetilde{\Lambda}_{2}$ in $\mathbb{R} P^{3}$, we obtain the linearized contact homology of $L(3,2)$ as

$$
\mathbb{H} C_{k}^{\operatorname{lin}}(L(3,2))= \begin{cases}\left\langle(a),\left(\widetilde{c}_{1}+c^{2} \widetilde{c}_{0}\right)\right\rangle & \text { for } k=1, \\ \left\langle\gamma^{k},\left(a^{k}\right),\left(\widetilde{c}_{1}+c^{2} \widetilde{c}_{0}\right)^{k}\right\rangle & \text { for } k>1 \text { odd }, \\ 0 & \text { otherwise. }\end{cases}
$$

One can use directly the cyclic Legendrian homology $L \mathbb{H}^{\mathrm{cyc}}\left(\Lambda_{1} \sqcup \Lambda_{2}\right)$ in (3) and the linearized contact homology of $S^{3}$ in the surgery exact sequence Theorem 1 for the surgery along $\Lambda_{1} \sqcup \Lambda_{2}$ in $S^{3}$ to obtain the linearized contact homology of $L(3,2)$.

\subsubsection{Linearized contact homology of the lens space $L(2 r+1,2)(r \geq 2)$ with its} different tight contact structures Let $\Lambda=\Lambda_{1} \sqcup \Lambda_{2}$ be a Legendrian link, where $\Lambda_{1}$ is the simple Legendrian unknot and $\Lambda_{2}$ is the Legendrian unknot with several right 
cusps and/or several left cusps in front projection. The Legendrian link under our consideration belongs to the following diagram. The lens space $L(2 r+1,2), r \geq 2$, with its different tight contact structures is obtained by the Legendrian surgery in $S^{3}$ along different Legendrian links that are shown in the $r^{\text {th }}$ row of this diagram (see Honda's Theorem 3).

$r=2$
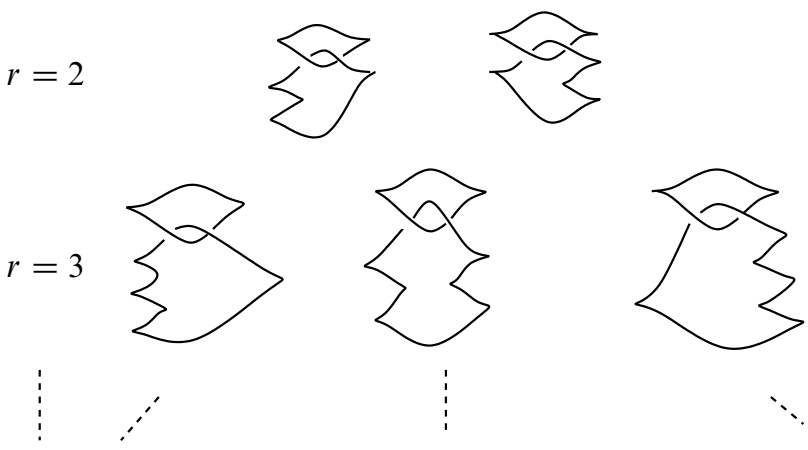

$\because$

Figure 9: Various Legendrian links in front projection

(1) Let us consider the case when the second component has several right cusps. We illustrate such a Legendrain link in the following figure in front projection.

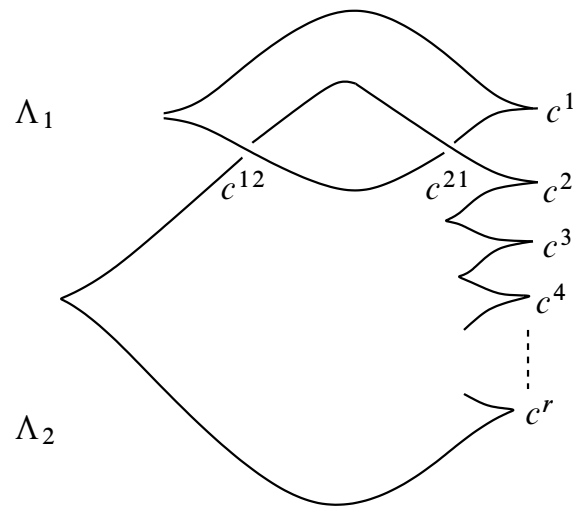

Figure 10: $\Lambda_{2}$ with several right cusps in front projection

The generators for the corresponding DGA are $c^{1}, \ldots, c^{r}$ and $c^{21}, c^{12}$ with the gradings

$$
\left|c^{1}\right|=\cdots=\left|c^{r}\right|=1, \quad\left|c^{21} c^{12}\right|=0,
$$

and the differential

$$
\partial c^{1}=1-1+c^{12} c^{21}, \quad \partial c^{2}=\cdots=\partial c^{r}=1, \quad \partial c^{21}=\partial c^{12}=0 .
$$


We perform Legendrian surgery along $\Lambda_{1}$. Hence, chords on $\tilde{\Lambda}_{2}$ are $c^{2}, \ldots, c^{r}$ and $\tilde{c}_{l}:=c^{21}\left(c^{1}\right)^{l} c^{12}, l \geq 0$ with the grading $\left|\widetilde{c}_{l}\right|=l$. The differentials of these generators are

$$
\partial c^{2}=\cdots=\partial c^{r}=1 \stackrel{\mathrm{cyc}}{=} 0, \quad \partial \widetilde{c}_{l}=\sum_{j=1}^{l}(-1)^{j} \widetilde{c}_{j-1} \widetilde{c}_{l-j} .
$$

It is easy to see that $L \mathbb{H}^{\text {cyc }}\left(\widetilde{\Lambda}_{2}\right)$ has one generator $\left(\widetilde{c}_{k}\right)$ when $k \neq 0$ is even and has one generator $\left(\left(c^{2}\right)^{k}\right)$ when $k \geq 1$ is odd. Using the surgery exact sequence for the surgery along $\widetilde{\Lambda}_{2}$ in $\mathbb{R} P^{3}$ and linearized contact homology of $\mathbb{R} P^{3}$ in (4) we obtain

$$
\mathbb{H} C_{k}^{\operatorname{lin}}\left(L(2 r+1,2), \xi_{1}\right)= \begin{cases}\left\langle(a),\left(c^{2}\right)\right\rangle & \text { for } k=1, \\ \left\langle\gamma^{k},\left(a^{k}\right),\left(\left(c^{2}\right)^{k}\right)\right\rangle & \text { for } k>1 \text { odd } \\ 0 & \text { otherwise }\end{cases}
$$

Here $\xi_{1}$ denotes the tight contact structure on the Lens space $L(2 r+1,2)$ obtained by Legendrian surgery along the above link.

(2) Consider the Legendrian link with the first component as the simple Legendrian unknot and the second component with several right cusps and several left cusps. In this case, the corresponding DGA is the same as the previous case. Therefore all computations and hence the linearized contact homology of the lens space $\left(L(2 r+1,2), \xi_{2}\right)$ obtained by surgery along such a link is the same as the previous case. That is,

$$
\mathbb{H} C_{k}^{\operatorname{lin}}\left(L(2 r+1,2), \xi_{2}\right)=\mathbb{H} C_{k}^{\operatorname{lin}}\left(L(2 r+1,2), \xi_{1}\right) .
$$

(3) Let the Legendrian link be with the first component as the simple Legendrian unknot and the second component with several left cusps. We illustrate such a Legendrain link in the following figure in front projection.

The generators of the DGA correspondence to this figure are right cusps $c^{1}, \ldots, c^{r}$, and $c^{21}, c^{12}$ with the gradings

$$
\left|c^{1}\right|=\cdots=\left|c^{r}\right|=1, \quad\left|c^{21} c^{12}\right|=0 .
$$

Moreover, their differentials are

$$
\begin{aligned}
\partial c^{1} & =1-1+c^{12} c^{21} \\
\partial c^{2} & =1+(-1)^{r-3} t c^{21} c^{12}\left(c^{3}\right)^{2} \cdots\left(c^{r}\right)^{2}+(-1)^{r-2} t\left(c^{3}\right)^{2} \cdots\left(c^{r}\right)^{2}, \\
\partial c^{3} & =\cdots=c^{r}=1, \\
\partial c^{21} & =\partial c^{12}=0 .
\end{aligned}
$$




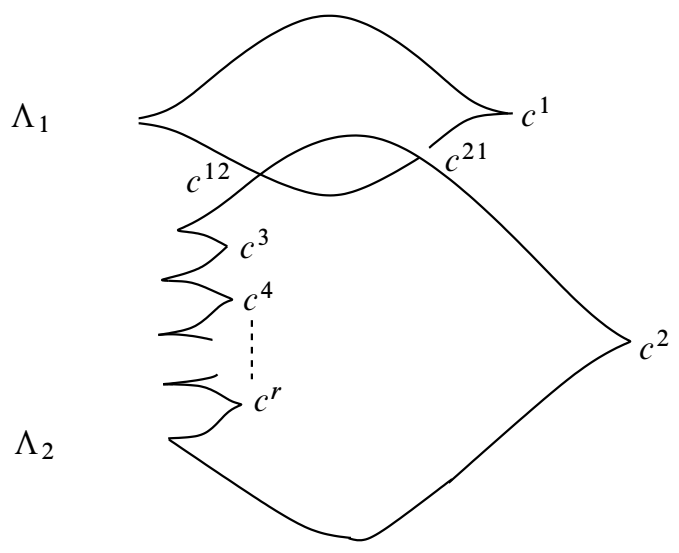

Figure 11: $\Lambda_{2}$ with several left cusps in front projection

Here we consider the DGA over $\mathbb{Q}\left[t, t^{-1}\right]$ where the indeterminate $t$ has degree $|t|=-2(r-2)$. We perform Legendrian surgery along $\Lambda_{1}$. Hence, chords on $\widetilde{\Lambda}_{2}$ are $c^{2}, \ldots, c^{r}$ and $\widetilde{c}_{l}:=c^{21}\left(c^{1}\right)^{l} c^{12}, l \geq 0$ with $\left|\widetilde{c}_{l}\right|=l$. The differentials of these generators are

$$
\begin{aligned}
& \partial c^{2} \stackrel{\text { cyc }}{=}(-1)^{r-3} t c^{21} c^{12}\left(c^{3}\right)^{2} \cdots\left(c^{r}\right)^{2}+(-1)^{r-2} t\left(c^{3}\right)^{2} \cdots\left(c^{r}\right)^{2}, \\
& \partial c^{3}=\cdots=\partial c^{r}=1 \stackrel{\text { cyc }}{=} 0, \\
& \partial \widetilde{c}_{l}=\sum_{j=1}^{l}(-1)^{j} \widetilde{c}_{j-1} \widetilde{c}_{l-j}
\end{aligned}
$$

We see that $L \mathbb{H}_{k}^{\mathrm{cyc}}\left(\widetilde{\Lambda}_{2}\right)$ has one generator $\left(\left(c^{3}\right)^{k}\right)$ for any odd degree $k \geq 1$, and has one generator $\left(\tilde{c}_{k}\right)$ for any even degree $k>0$. Then we use the surgery exact sequence for the surgery along $\widetilde{\Lambda}_{2}$ in $\mathbb{R} P^{3}$. The linearized contact homology of $\left(L(2 r+1,2), \xi_{3}\right)$ obtained by the Legendrian surgery along the above link is (as a module over $\mathbb{Q}$ )

$$
\mathbb{H} C_{k}^{\operatorname{lin}}\left(L(2 r+1,2), \xi_{3}\right)= \begin{cases}\left\langle(a),\left(c^{3}\right)\right\rangle & \text { for } k=1 \\ \left\langle\gamma^{k},\left(a^{k}\right),\left(\left(c^{3}\right)^{k}\right)\right\rangle & \text { for } k>1 \text { odd } \\ 0 & \text { otherwise }\end{cases}
$$

Note that in these three cases one can compute the cyclic Legendrian homology $L \mathbb{H}^{\text {cyc }}\left(\Lambda_{1} \sqcup \Lambda_{2}\right)$ using the exact sequence of Theorem 2 . Then one can use the surgery exact sequence of Theorem 1 for the surgery along $\Lambda_{1} \sqcup \Lambda_{2}$ in $S^{3}$ to obtain the linearized contact homologies of $L(2 r+1,2)$. 
These computations show that the linearized contact homologies of the lens space $L(2 r+1,2), r \geq 2$, with its all tight contact structures are the same and the linearized contact homology is not a complete invariant to distinguish them.

\subsection{Standard Legendrian Hopf link in $S^{2 n-1}$}

Let $\Lambda=\Lambda_{1} \sqcup \Lambda_{2}$ be the standard Legendrian Hopf link, shown in Figure 12, in the contact manifold $Y_{0}=S^{2 n-1}$ equipped with the standard contact structure. We equip $Y_{0}$ with a contact form corresponding to an ellipsoid $S^{2 n-1} \subset \mathbb{R}^{2 n}$ with rationally independent axis lengths, so that the Reeb field has only finitely many closed orbits. After a Legendrian isotopy, we can always assume that $\Lambda$ is contained in a small Darboux ball disjoint from the closed Reeb orbits. By shrinking the size of this ball, we can always arrange so that the return time of the Reeb flow to this ball is as large as desired. In particular, all Reeb chords of $\Lambda$ that are not contained in the Darboux ball will be arbitrarily long. In view of the action filtration process described in [4], we can ignore these chords. Therefore, we can concentrate only on the Darboux ball containing $\Lambda$, so that our computations will be the same as if $\Lambda$ was a Legendrian link in $\mathbb{R}^{2 n-1}$ with the standard contact structure. This link is represented on Figure 12.

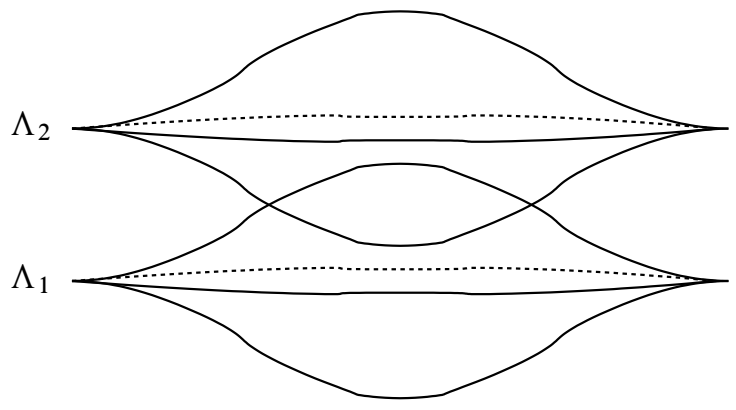

Figure 12: Standard Legendrian Hopf link

This Legendrian link contains degenerate Reeb chords, since every point of the bottom component $\Lambda_{1}$ is the origin of a chord ending on the top component $\Lambda_{2}$ after a short flow time. In order to make all chords generic, we choose to slightly tilt one of the components by adding a small linear function, say of the $x_{1}$-coordinate, to its front projection. There are 6 chords for this Legendrian link: one between the two branches of $\Lambda_{1}$, denoted by $c^{1}$, one between the two branches of $\Lambda_{2}$, denoted by $c^{2}$, two between the top branch of $\Lambda_{2}$ and the bottom branch of $\Lambda_{1}$, and two between the bottom branch of $\Lambda_{2}$ and the top branch of $\Lambda_{1}$. We denote by $c^{12}$ and by $c^{21}$ the long chords from $\Lambda_{1}$ to $\Lambda_{2}$ and from $\Lambda_{2}$ to $\Lambda_{1}$ respectively. The short chords 
from $\Lambda_{2}$ to $\Lambda_{1}$ will be denoted by $c_{m}$ and $c_{M}$, corresponding to the smallest and to the largest value of the action respectively. These 6 chords are the edges of a quiver in Figure 13 whose vertices are $\Lambda_{1}$ and $\Lambda_{2}$. The orientation of the edges corresponds to the direction of the Reeb field.

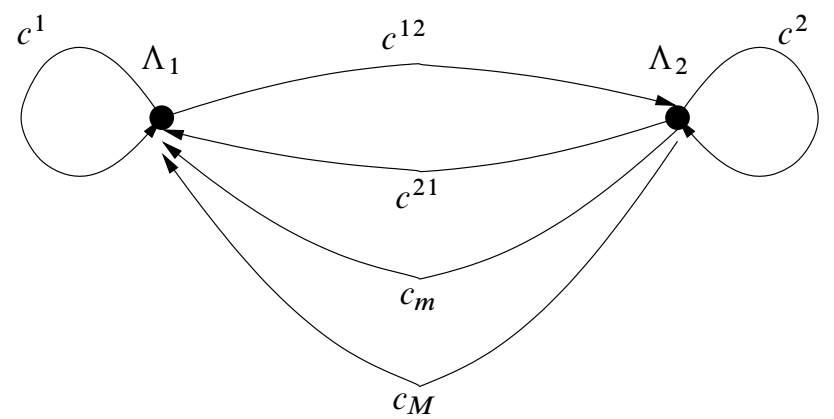

Figure 13: Reeb chords of $\Lambda$

Using the index formula in front projection given in [10, Lemma 3.4], the grading of $c^{1}$ and $c^{2}$ can be computed easily with each component separately, since it corresponds to a maximum of the height function and going down a cusp: $\left|c^{1}\right|=\left|c^{2}\right|=n-1$. The grading of the mixed chords is defined only modulo an additive constant. For convenience, we set this constant (denoted by $k$ ) to be the grading of the chord $c_{m}$. The grading of $c_{M}$ can be obtained by adding to $\left|c_{m}\right|$ the Morse index of the maximum on $S^{n-1}$. The grading of $c^{21}$ differs from the grading of $c_{M}$ by the contribution of going up a cusp. Finally, the grading of $c^{12}$ is obtained by reversing the sign of the constant and going down a cusp instead of going up a cusp. We therefore obtain

$$
\begin{aligned}
\left|c^{1}\right| & =n-1, \\
\left|c^{2}\right| & =n-1, \\
\left|c^{21}\right| & =n+k, \\
\left|c^{12}\right| & =n-2-k, \\
\left|c_{M}\right| & =n-1+k, \\
\left|c_{m}\right| & =k .
\end{aligned}
$$

3.3.1 Holomorphic curves via Morse flow trees In order to find the rigid holomorphic curves that appear in the differential for Legendrian contact homology of $\Lambda$, it will be necessary to use the bijective correspondence between those holomorphic discs with the Morse flow trees developed by Ekholm [8]. 
Given a metric $g$ on $\mathbb{R}^{n-1}$, a flow tree on $\Lambda \subset J^{1}\left(\mathbb{R}^{n-1}\right)$ is a finite tree $\Gamma$ immersed by $f: \Gamma \rightarrow \mathbb{R}^{n-1}$, together with extra data, such that:

(a) On the interior of an edge $e_{i}, f$ is an injective parametrization of a flow line of $-\nabla\left(h_{i}^{\alpha}-h_{i}^{\beta}\right)$, where $h_{i}^{\alpha}$ and $h_{i}^{\beta}$ are two locally defined real-valued functions, each defining the $z$-coordinate of a sheet of $\Lambda$. To the flow line corresponding to $e_{i}$ we associate its two 1 -jet lifts $\phi_{i}^{\alpha}, \phi_{i}^{\beta}$, parameterized by

$$
\begin{aligned}
& \phi_{i}^{\alpha}(t)=\left(d h_{i}^{\alpha}\left(e_{i}(t)\right), h_{i}^{\alpha}\left(e_{i}(t)\right)\right) \in \Lambda \subset J^{1}\left(\mathbb{R}^{n-1}\right)=\mathbb{R}^{2 n-1}, \\
& \phi_{i}^{\beta}(t)=\left(d h_{i}^{\beta}\left(e_{i}(t)\right), h_{i}^{\beta}\left(e_{i}(t)\right)\right) \in \Lambda \subset J^{1}\left(\mathbb{R}^{n-1}\right)=\mathbb{R}^{2 n-1},
\end{aligned}
$$

and oriented by $-\nabla\left(h_{i}^{\alpha}-h_{i}^{\beta}\right)$ and $-\nabla\left(h_{i}^{\beta}-h_{i}^{\alpha}\right)$, respectively.

(b) For every vertex $v$ we fix a cyclic ordering of the edges $\left\{e_{i}\right\}$. We denote the unique 1 -jet lift of the $i^{\text {th }}$ edge which is oriented towards (away from) the vertex $v$ by $\phi_{i}^{\text {in }, v}\left(\phi_{i}^{\text {out }, v}\right)$.

(c) Consider the curves on $\Lambda \subset J^{1}\left(\mathbb{R}^{n-1}\right)$ given by the oriented 1-jet lifts of the flow lines. Give the curves a cyclic order by declaring that for every vertex $v$ and edge $i$, the curve $\phi_{i}^{\text {in }, v}$ is succeeded by $\phi_{i+1}^{\text {out } v}$. We require that the Lagrangian projections of the oriented 1 -jet lifts in this order form a closed curve on the projection of $\Lambda$ in $T^{*} \mathbb{R}^{n-1}$.

If the 1 -jet lifts $\phi_{i}^{\text {in, } v}$ and $\phi_{i+1}^{\text {out }, v}$ have different values at the vertex $v$, we call this a puncture at the vertex. The puncture is called positive if the oriented curve jumps from a lower to a higher sheet relative the $z$-coordinate, and is otherwise called negative.

The various types of vertices that can appear in a flow tree are listed in Figure 14, borrowed from Dimitroglou Rizell [7]. In that figure, (P1) and (P2) depict the punctures in the generic case, (E) and (S) depict the vertices corresponding to an end and a switch, respectively, while (Y0) and (Y1) describes the generic 3-valent vertices.

Because of the following result, we may use rigid flow trees to compute the Legendrian contact homology.

Theorem 5 [8, Theorem 1.1] For a generic perturbation of $\Lambda$ and the metric $g$ on $\mathbb{R}^{n-1}$, there is a regular almost complex structure $J$ on $T^{*} \mathbb{R}^{n-1}$ such that there is a bijective correspondence between rigid $J$-holomorphic discs with one positive puncture having boundary on a perturbation of $\Lambda$, and rigid flow trees on $\Lambda$ with one positive puncture. 


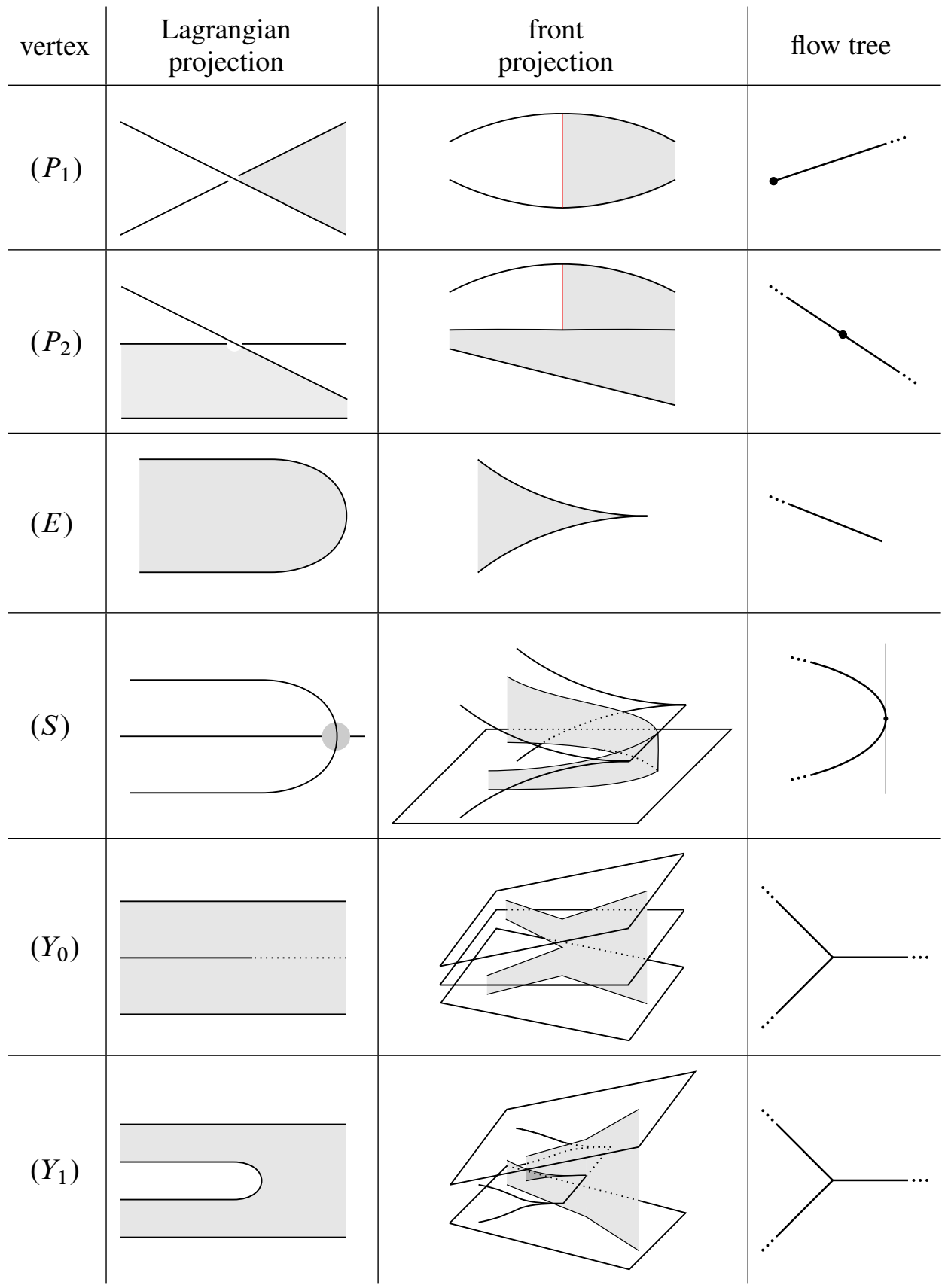

Figure 14: Possible vertices in a flow tree 
In order to apply the above theorem, we have to perturb $\Lambda$ by translating the component $\Lambda_{1}$ along a horizontal direction (in its front projection), say the $x_{1}$-axis. After this perturbation, the 6 chords of $\Lambda$ have different $x_{1}$-coordinates.

Let us first compute the differential of the shortest chords $c_{m}$ and $c_{M}$. Since the differential decreases the action, the differential of $c_{m}$ has to vanish and the differential of $c_{M}$ has to be a multiple of $c_{m}$. Since $\left|c_{M}\right|=\left|c_{m}\right|+n-1$, the differential of $c_{M}$ has to vanish when $n>2$. If $n=2$, Theorem 5 shows that the latter differential counts flow trees from $c_{M}$ to $c_{m}$. Each such flow tree consists of a segment with 2 vertices of type (E) at its endpoints and two internal vertices of type (P2), corresponding to $c_{M}$ and to $c_{m}$ respectively, as illustrated on Figure 15. This figure shows the Legendrian link after perturbation in the $y$ direction in Lagrangian projection. The 2 vertices of type (E) has to correspond to a cusp of $\Lambda_{1}$ and to a cusp of $\Lambda_{2}$, but can be interchanged. Therefore there are exactly two such flow trees that are counted with opposite signs (we are using the combinatorial signs from Etnyre, $\mathrm{Ng}$ and Sabloff [13] for two holomorphic discs which are, by Theorem 5, in correspondence with these two flow trees), so that they cancel each other. This coincides with the fact that the Morse differential for a perfect Morse function on $S^{1}$ vanishes.

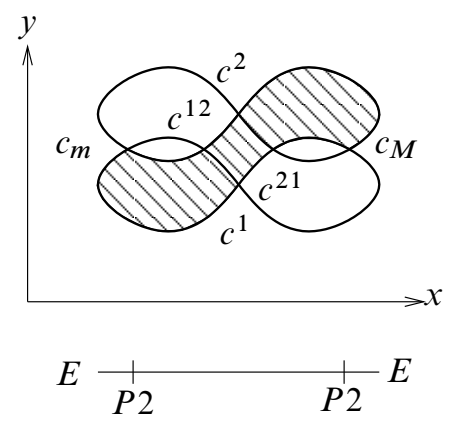

Figure 15: Rigid flow trees from $c_{M}$ to $c_{m}$ when $n=2$

Then the differential of $c^{12}$ has to consist of a linear combination of words of chords that constitute a path from $\Lambda_{1}$ to $\Lambda_{2}$ on the quiver of Figure 13. Since any such word has to contain the letter $c^{12}$, it follows that the action of such a word is at least equal to the action of $c^{12}$. Since the differential decreases the action, it follows that $\partial c^{12}=0$.

Next the differential of $c^{1}$ has to consist of a linear combination of words of chords that constitute a loop based at $\Lambda_{1}$. Since $c^{1}$ and its iterates are forbidden for action reasons, this loop has to contain $c^{12}$. It therefore cannot contain $c^{2}, c^{21}$ or another copy of $c^{12}$ as well, again for action reasons. Therefore, the possible words are $c^{12} c_{m}$ and $c^{12} c_{M}$. Since the latter has grading $2 n-3 \geq n-1=\left|c^{1}\right|$, this word is forbidden as 
well, so that the differential of $c^{1}$ is a multiple of $c^{12} c_{m}$. By Theorem 5, this multiple counts the flow trees starting at $c^{1}$ and going along the $x_{1}$-axis towards $c^{12}$. The tree then continues along the $x_{1}$-axis to $c_{m}$, and then ends at a cusp. The corresponding tree consists of a segment with a vertex of type (P1) at one endpoint, corresponding to $c^{1}$, two internal vertices of type (P2), corresponding to $c^{12}$ and to $c_{m}$, and a vertex of type (E) at the other endpoint. Since there is a unique such flow tree, we obtain $\partial c^{1}= \pm c^{12} c_{m}$. Similarly, we have $\partial c^{2}= \pm c_{m} c^{12}$.

Note that when $n=2$ we have additional terms in the differential of $c^{1}$ and of $c^{2}$. These terms correspond to the flow trees which start and end at $c^{1}$ (at $c^{2}$ ) going along $x_{1}$-axis without any vertex. Therefore we have $\partial c^{1}= \pm 1 \mp 1 \pm c^{12} c_{m}= \pm c^{12} c_{m}$ and $\partial c^{2}= \pm 1 \mp 1 \pm c_{m} c^{12}= \pm c_{m} c^{12}$. Here we used the combinatorial signs from [13] for the two additional terms with opposite signs in $\partial c^{1}$ and similarly in $\partial c^{2}$.

Finally, the differential of $c^{21}$ has to consist of a linear combination of words of chords that constitute a path from $\Lambda_{2}$ to $\Lambda_{1}$ on the quiver of Figure 13. It can either consist of $c_{m}$ or of $c_{M}$, multiplied with copies of $c^{1}$ on the right and $c^{2}$ on the left, or contain at least a copy of $c^{12}$. In the former case, for action reasons, there can be at most a copy of $c^{1}$ or $c^{2}$, so the possibilities are $c_{p}, c_{p} c^{1}$ and $c^{2} c_{p}$ with $p=m, M$. Note that $\left|c_{m}\right|=k<\left|c^{21}\right|-1$ so that $c_{m}$ is forbidden. Note that $\left|c_{M} c^{1}\right|=\left|c^{2} c_{M}\right|=2 n-2+k \geq\left|c^{21}\right|$ so that $c_{M} c^{1}$ and $c^{2} c_{M}$ are forbidden. In the latter case, for action reasons, this word cannot contain $c^{1}, c^{2}, c^{21}$ or another copy of $c^{12}$. It could therefore be $c_{p} c^{12} c_{q}$ with $p, q=m, M$. Note that $\left|c_{m} c^{12} c_{m}\right|=$ $n-2+k<\left|c^{21}\right|-1$, that $\left|c_{m} c^{12} c_{M}\right|=\left|c_{m} c^{12} c_{M}\right|=2 n-3+k=\left|c^{21}\right|+n-3$ and that $\left|c_{M} c^{12} c_{M}\right|=3 n-4+k \geq\left|c^{21}\right|$, so that $c_{m} c^{12} c_{m}, c_{M} c^{12} c_{M}$ are always forbidden and $c_{m} c^{12} c_{M}, c_{M} c^{12} c_{m}$ are forbidden when $n>2$. Therefore, when $n>2, \partial c^{21}$ is a linear combination of $c_{M}, c_{m} c^{1}$ and $c^{2} c_{m}$. By Theorem 5, the coefficients count the flow trees starting at $c^{21}$ and going along the $x_{1}$-axis towards $c_{M}, c^{1}$ and $c^{2}$ respectively. In the first case, the tree consists of a segment along the $x_{1}$-axis with vertices of type (P1) at its endpoints, corresponding to $c^{21}$ and to $c_{M}$. In the last two cases, the tree is a segment along the $x_{1}$-axis with a vertex of type (P1) at one endpoints, corresponding to $c^{21}$, two intermediate vertices of type (P2), corresponding to $c_{m}$ and $c^{1}$, or to $c^{2}$ and $c_{m}$, and a vertex of type (E) at the other endpoint. There is always one such flow tree, so that $\partial c^{21}= \pm c_{M} \pm c_{m} c^{1} \pm c^{2} c_{m}$ when $n>2$. If $n=2$, enumerating immersed polygons in the Lagrangian projection of $\Lambda$ as in [6] shows that these are all the terms in the differential.

In summary, the differential for the Legendrian contact homology of $\Lambda$ has the form

$$
\begin{aligned}
& \partial c_{M}=0, \\
& \partial c_{m}=0, \\
& \partial c^{12}=0, \\
& \partial c^{21}= \pm c_{M} \pm c_{m} c^{1} \pm c^{2} c_{m}, \\
& \partial c^{1}= \pm c^{12} c_{m}, \\
& \partial c^{2}= \pm c_{m} c^{12} \text {. }
\end{aligned}
$$


Note that the first four undetermined signs in the above equations can be absorbed in the definition of $c^{21}, c^{1}, c^{2}$ and $c^{12}$ respectively. On the other hand, since $\partial^{2} c^{21}=0$, using the Leibniz rule we must have

$$
\begin{aligned}
0 & =\partial c_{M}+\left(\partial c_{m}\right) c^{1}+(-1)^{\left|c_{m}\right|} c_{m} \partial c^{1}+\left(\partial c^{2}\right) c_{m}+(-1)^{\left|c^{2}\right|} c^{2} \partial c_{m} \\
& =(-1)^{k} c_{m} c^{12} c_{m} \pm c_{m} c^{12} c_{m}
\end{aligned}
$$

so that the last sign is necessarily $(-1)^{k+1}$. Therefore, the differential for the Legendrian contact homology of $\Lambda$ is given by

$$
\begin{aligned}
& \partial c_{M}=0, \quad \partial c_{m}=0, \quad \partial c^{12}=0, \\
& \partial c^{21}=c_{M}+c_{m} c^{1}+c^{2} c_{m}, \quad \partial c^{1}=c^{12} c_{m}, \quad \partial c^{2}=(-1)^{k+1} c_{m} c^{12} .
\end{aligned}
$$

3.3.2 Legendrian homology after surgery After performing Legendrian surgery along $\Lambda_{1}$, we obtain the contact manifold $Y_{1}$ containing a copy $\widetilde{\Lambda}_{2}$ of the other component of $\Lambda$. Here we would like to describe the cyclic Legendrian homology of $\tilde{\Lambda}_{2}$. We recall that the module $\operatorname{LH}^{\text {cyc }}\left(\tilde{\Lambda}_{2}\right)$ is generated by the cyclic words in chords of $\tilde{\Lambda}_{2}$ in which the chords have two types: the chords of $\Lambda_{2}$ unaffected by the surgery along $\Lambda_{1}$ and the chords created by this surgery. A simple inspection of the quiver on Figure 13 shows that the created chords are presented (by Proposition 1) in the words of the form $c^{21}\left(c^{1}\right)^{l} c^{12}, c_{m}\left(c^{1}\right)^{l} c^{12}$ or $c_{M}\left(c^{1}\right)^{l} c^{12}$, for any integer $l \geq 0$. We will denote the corresponding chords of $\tilde{\Lambda}_{2}$ by $\widetilde{c}_{l}, \widetilde{m}_{l}$ and $\tilde{M}_{l}$ respectively. Their gradings are given by

$$
\left|\tilde{c}_{l}\right|=2 n-2+l(n-1), \quad\left|\tilde{m}_{l}\right|=n-2+l(n-1), \quad\left|\tilde{M}_{l}\right|=2 n-3+l(n-1) .
$$

The differential for the Legendrian contact homology of $\widetilde{\Lambda}_{2}$ is induced by the differential computed in the previous section:

$$
\begin{aligned}
\partial \widetilde{c}_{l} & \equiv \partial\left(c^{21}\left(c^{1}\right)^{l} c^{12}\right) \\
& =\left(c_{M}+c_{m} c^{1}+c^{2} c_{m}\right)\left(c^{1}\right)^{l} c^{12}+\sum_{i=1}^{l} c^{21}\left(c^{1}\right)^{i-1} c^{12} c_{m}\left(c^{1}\right)^{l-i} c^{12} \\
& =c_{M}\left(c^{1}\right)^{l} c^{12}+c_{m}\left(c^{1}\right)^{l+1} c^{12}+c^{2}\left(c_{m}\left(c^{1}\right)^{l} c^{12}\right) \\
& +\sum_{i=1}^{l}\left(c^{21}\left(c^{1}\right)^{i-1} c^{12}\right)\left(c_{m}\left(c^{1}\right)^{l-i} c^{12}\right) \\
& \equiv \tilde{M}_{l}+\tilde{m}_{l+1}+c^{2} \tilde{m}_{l}+\sum_{i=1}^{l} \tilde{c}_{i-1} \tilde{m}_{l-i},
\end{aligned}
$$




$$
\begin{aligned}
\partial \tilde{m}_{l} \equiv \partial\left(c_{m}\left(c^{1}\right)^{l} c^{12}\right) & =\sum_{i=1}^{l} c_{m}\left(c^{1}\right)^{i-1} c^{12} c_{m}\left(c^{1}\right)^{l-i} c^{12} \\
& =\sum_{i=1}^{l}\left(c_{m}\left(c^{1}\right)^{i-1} c^{12}\right)\left(c_{m}\left(c^{1}\right)^{l-i} c^{12}\right) \equiv \sum_{i=1}^{l} \tilde{m}_{i-1} \tilde{m}_{l-i} \\
\partial \tilde{M}_{l} \equiv \partial\left(c_{M}\left(c^{1}\right)^{l} c^{12}\right) & =\sum_{i=1}^{l} c_{M}\left(c^{1}\right)^{i-1} c^{12} c_{m}\left(c^{1}\right)^{l-i} c^{12} \\
& =\sum_{i=1}^{l}\left(c_{M}\left(c^{1}\right)^{i-1} c^{12}\right)\left(c_{m}\left(c^{1}\right)^{l-i} c^{12}\right) \equiv \sum_{i=1}^{l} \tilde{M}_{i-1} \tilde{m}_{l-i}
\end{aligned}
$$

and

$$
\partial c^{2} \equiv(-1)^{k+1} c_{m} c^{12} \equiv(-1)^{k+1} \tilde{m}_{0}
$$

The differential for the Legendrian contact homology of $\widetilde{\Lambda}_{2}$ is therefore given by

$$
\begin{array}{rlrl}
\partial \widetilde{c}_{l} & =\tilde{M}_{l}+\tilde{m}_{l+1}+c^{2} \tilde{m}_{l}+\sum_{i=1}^{l} \tilde{c}_{i-1} \tilde{m}_{l-i}, & \partial \tilde{m}_{l}=\sum_{i=1}^{l} \tilde{m}_{i-1} \tilde{m}_{l-i}, \\
\partial \tilde{M}_{l}=\sum_{i=1}^{l} \tilde{M}_{i-1} \tilde{m}_{l-i}, & \partial c^{2}=(-1)^{k+1} \tilde{m}_{0} .
\end{array}
$$

The module $\operatorname{LH}^{\mathrm{cyc}}\left(\tilde{\Lambda}_{2}\right)$ is generated by cyclic words in the letters $\widetilde{c}_{l}, \tilde{m}_{l}, \tilde{M}_{l}(l \geq 0)$ and $c^{2}$, with the induced differential.

As a comparison, the module $\mathrm{LH}^{\text {cyc }}(\Lambda)$ is generated by composable words up to cyclic permutations in the letters $c^{1}, c^{2}, c^{12}, c^{21}, c_{m}$ and $c_{M}$. Such composable words up to cyclic permutation correspond to unbased loops in the quiver of Figure 13. The direct computation of $L \mathbb{H}^{\text {cyc }}(\Lambda)$ via these words seems to be quite untractible. On the other hand, the computation of $L \mathbb{H}^{\mathrm{cyc}}\left(\tilde{\Lambda}_{2}\right)$ using the above chain complex seems possible. Combining this computation with the exact sequence of Theorem 2, we obtain a realistic way of computing the invariant $L \mathbb{H}^{\text {cyc }}(\Lambda)$.

Remark 6 When $n=2$ we can use the first Legendrian Reidemeister move to cancel two generators $c_{M}$ and $c^{21}$ in Figure 15. Then we are in the situation of Section 3.1 with four generators $c^{1}, c^{2}, c^{12}, c_{m}$ (here $c_{m}$ corresponds to $c^{21}$ with the notation of the Section 3.1). The gradings are $\left|c^{1}\right|=\left|c^{2}\right|=1$ and $\left|c_{m} c^{12}\right|=0$. The module $\operatorname{LH}^{\text {cyc }}\left(\tilde{\Lambda}_{2}\right)$ is generated by $\tilde{m}_{l}$ for $l \geq 0$ and $c^{2}$. 
Remark 7 Now consider $n=3$. Here we are going to compute $L \mathbb{H}^{\text {cyc }}\left(\tilde{\Lambda}_{2}\right)$ and $L \mathbb{H}^{\text {cyc }}\left(\Lambda_{1} \sqcup \Lambda_{2}\right)$ for some low degrees. The Reeb chords on $\widetilde{\Lambda}_{2}$ are $c^{2}$ and $\widetilde{c}_{l}, \widetilde{m}_{l}, \widetilde{M}_{l}$ for $l \geq 0$. Their gradings are given by

$$
\left|c^{2}\right|=2, \quad\left|\tilde{c}_{l}\right|=4+2 l, \quad\left|\tilde{m}_{l}\right|=1+2 l, \quad\left|\tilde{M}_{l}\right|=3+2 l .
$$

Then we have the following cyclic complex:

$$
\begin{aligned}
& \operatorname{LH}_{0}^{\text {cyc }}\left(\tilde{\Lambda}_{2}\right)=0 \\
& \operatorname{LH}_{1}^{\text {cyc }}\left(\tilde{\Lambda}_{2}\right)=\left\langle\left(\tilde{m}_{0}\right)\right\rangle \\
& \operatorname{LH}_{2}^{\text {cyc }}\left(\tilde{\Lambda}_{2}\right)=\left\langle\left(c^{2}\right)\right\rangle \\
& \operatorname{LH}_{3}^{\text {cyc }}\left(\tilde{\Lambda}_{2}\right)=\left\langle\left(\tilde{m}_{0} c^{2}\right),\left(\tilde{m}_{1}\right),\left(\tilde{m}_{0}^{3}\right),\left(\tilde{M}_{0}\right)\right\rangle \\
& \operatorname{LH}_{4}^{\mathrm{cyc}}\left(\tilde{\Lambda}_{2}\right)=\left\langle\left(\tilde{c}_{0}\right),\left(\left(c^{2}\right)^{2}\right),\left(c^{2} \tilde{m}_{0}^{2}\right),\left(\tilde{M}_{0} \tilde{m}_{0}\right)\right\rangle
\end{aligned}
$$

Since there is no generator in degree zero, we have $L \mathbb{H}_{0}^{\text {cyc }}\left(\tilde{\Lambda}_{2}\right)=0$. In degree one, $\partial\left(\tilde{m}_{0}\right)=\partial\left(c_{m} c^{12}\right)=0$. On the other hand $\partial\left(c^{2}\right)=(-1)^{k+1} \tilde{m}_{0}$. Therefore, $L \mathbb{H}_{1}^{\text {cyc }}\left(\widetilde{\Lambda}_{2}\right)=0$. In degree two, $L \mathbb{H}_{2}^{\text {cyc }}\left(\widetilde{\Lambda}_{2}\right)=0$ because $\partial\left(c^{2}\right)$ is nonzero. In degree three, the differential is zero. We have

$$
\partial\left(\tilde{m}_{0} c^{2}\right)=(-1)^{k} \tilde{m}_{0}^{2} \stackrel{\text { cyc }}{=} 0, \quad \partial\left(\tilde{m}_{1}\right)=\partial\left(c_{m} c^{1} c^{12}\right)=(-1)^{k} \tilde{m}_{0}^{2} \stackrel{\text { cyc }}{=} 0,
$$

( $\tilde{m}_{0}^{2}$ is bad), and also

$$
\partial\left(\tilde{m}_{0}^{3}\right)=0, \quad \partial\left(\tilde{M}_{0}\right)=\partial\left(c_{M} c^{12}\right)=0 .
$$

On the other hand we have

$$
\begin{aligned}
\partial\left(\tilde{c}_{0}\right) & =\partial\left(c^{21} c^{12}\right)=\tilde{M}_{0}+\tilde{m}_{1}+c^{2} \tilde{m}_{0}, \\
\partial\left(\left(c^{2}\right)^{2}\right) & \stackrel{\text { cyc }}{=} 2(-1)^{k+1} c^{2} \tilde{m}_{0}, \\
\partial\left(c^{2} \tilde{m}_{0}^{2}\right) & =(-1)^{k+1} \tilde{m}_{0}^{3}, \\
\partial\left(\tilde{M}_{0} \tilde{m}_{0}\right) & =0 .
\end{aligned}
$$

Therefore $L \mathbb{H}_{3}^{\mathrm{cyc}}\left(\tilde{\Lambda}_{2}\right)=\left\langle\left(\tilde{m}_{1}\right)\right\rangle$. In degree four $L \mathbb{H}_{4}^{\mathrm{cyc}}\left(\tilde{\Lambda}_{2}\right)=0$, since $\partial\left(\tilde{M}_{1}\right)=$ $(-1)^{k+2} \tilde{M}_{0} \tilde{m}_{0}$.

Now consider $\Lambda_{1}$ with its only Reeb chord $a$ of grading $|a|=1$ with $\partial a=0$ (see [4]). $L \mathbb{H}^{\text {cyc }}\left(\Lambda_{1}\right)$ is zero in even degrees, and is equal to $\left\langle\left(a^{2 l+1}\right) \mid l \geq 0\right\rangle$ in odd degrees. Using the exact sequence of Theorem 2 we obtain that $L \mathbb{H}^{\text {cyc }}\left(\Lambda_{1} \sqcup \Lambda_{2}\right)$ is zero in degrees $0,2,4$ and is equal to $\langle(a)\rangle,\left\langle\left(\tilde{m}_{1}\right)\right\rangle \oplus\left\langle\left(a^{3}\right)\right\rangle$ in degrees 1,3 respectively. 
Remark 8 When $n>3$ the cyclic complex is as follows:

$$
\begin{aligned}
\operatorname{LH}_{n-2}^{\mathrm{cyc}}\left(\tilde{\Lambda}_{2}\right) & =\left\langle\left(\tilde{m}_{0}\right)\right\rangle \\
\operatorname{LH}_{n-1}^{\mathrm{cyc}}\left(\tilde{\Lambda}_{2}\right) & =\left\langle\left(c^{2}\right)\right\rangle \\
\operatorname{LH}_{2 n-3}^{\mathrm{cyc}}\left(\tilde{\Lambda}_{2}\right) & =\left\langle\left(\tilde{M}_{0}\right),\left(\tilde{m}_{1}\right),\left(c^{2} \tilde{m}_{0}\right)\right\rangle
\end{aligned}
$$

We obtain

$$
\begin{aligned}
L \mathbb{H}_{n-3}^{\mathrm{cyc}}\left(\tilde{\Lambda}_{2}\right) & =0, \\
L \mathbb{H}_{n-1}^{\mathrm{cyc}}\left(\tilde{\Lambda}_{2}\right) & =0, \\
L \mathbb{H}_{2 n-3}^{\mathrm{cyc}}\left(\tilde{\Lambda}_{2}\right) & =\left\langle\left(\tilde{m}_{1}\right)\right\rangle \\
L \mathbb{H}_{2 n-3}^{\mathrm{cyc}}\left(\tilde{\Lambda}_{2}\right) & =\left\langle\left(\tilde{m}_{1}\right),\left(c^{2} \tilde{m}_{0}\right)\right\rangle \quad \text { when } n \text { is odd } \\
&
\end{aligned}
$$

Acknowledgements This work is based on my $\mathrm{PhD}$ thesis under the supervision of Frederic Bourgeois. I would like to thank him for introducing me to the world of contact homology, and for his comments and advice. I also would like to thank the anonymous referee for the very useful comments and suggestions that helped me to improve the manuscript. My research was supported by a graduate fellowship from Université Libre de Bruxelles. I also benefited the support of CAST, a Research Network Program of the European Science Foundation.

\section{References}

[1] F Bourgeois, Introduction to contact homology, notes from a summer school in Berder: Holomorphic curves and contact topology (2003)

[2] F Bourgeois, A survey of contact homology, from: "New perspectives and challenges in symplectic field theory", (M Abreu, F Lalonde, L Polterovich, editors), CRM Proc. Lecture Notes 49, Amer. Math. Soc. (2009) 45-71 MR2555933

[3] F Bourgeois, K Cieliebak, T Ekholm, A note on Reeb dynamics on the tight 3-sphere, J. Mod. Dyn. 1 (2007) 597-613 MR2342700

[4] F Bourgeois, T Ekholm, Y Eliashberg, Effect of Legendrian surgery, Geom. Topol. 16 (2012) 301-389 MR2916289

[5] F Bourgeois, Y Eliashberg, H Hofer, K Wysocki, E Zehnder, Compactness results in symplectic field theory, Geom. Topol. 7 (2003) 799-888 MR2026549

[6] Y Chekanov, Differential algebra of Legendrian links, Invent. Math. 150 (2002) 441483 MR1946550 
[7] G Dimitroglou Rizell, Knotted Legendrian surfaces with few Reeb chords, Algebr. Geom. Topol. 11 (2011) 2903-2936 MR2846915

[8] T Ekholm, Morse flow trees and Legendrian contact homology in 1-jet spaces, Geom. Topol. 11 (2007) 1083-1224 MR2326943

[9] T Ekholm, J Etnyre, M Sullivan, The contact homology of Legendrian submanifolds in $\mathbb{R}^{2 n+1}$, J. Differential Geom. 71 (2005) 177-305 MR2197142

[10] T Ekholm, J Etnyre, M Sullivan, Nonisotopic Legendrian submanifolds in $\mathbb{R}^{2 n+1}$, J. Differential Geom. 71 (2005) 85-128 MR2191769

[11] T Ekholm, J Etnyre, M Sullivan, Orientations in Legendrian contact homology and exact Lagrangian immersions, Internat. J. Math. 16 (2005) 453-532 MR2141318

[12] Y Eliashberg, A Givental, H Hofer, Introduction to symplectic field theory, Geom. Funct. Anal. (2000) 560-673 MR1826267

[13] J B Etnyre, L L Ng, J M Sabloff, Invariants of Legendrian knots and coherent orientations, J. Symplectic Geom. 1 (2002) 321-367 MR1959585

[14] H Geiges, An introduction to contact topology, Cambridge Studies in Adv. Math. 109, Cambridge Univ. Press (2008) MR2397738

[15] K Honda, On the classification of tight contact structures, I, Geom. Topol. 4 (2000) 309-368 MR1786111

[16] D McDuff, D Salamon, J-holomorphic curves and symplectic topology, Amer. Math. Soc. Coll. Publ. 52, Amer. Math. Soc. (2004) MR2045629

[17] L L Ng, Computable Legendrian invariants, Topology 42 (2003) 55-82 MR1928645

[18] J Robbin, D Salamon, The Maslov index for paths, Topology 32 (1993) 827-844 MR1241874

[19] P Seidel, A long exact sequence for symplectic Floer cohomology, Topology 42 (2003) 1003-1063 MR1978046

[20] A Weinstein, Contact surgery and symplectic handlebodies, Hokkaido Math. J. 20 (1991) 241-251 MR1114405

Département de Mathématiques, Université Libre de Bruxelles

CP 218 Boulevard du Triomphe, 1050 Bruxelles, Belgium

aeslamir@ulb.ac.be

Received: 18 November $2013 \quad$ Revised: 15 June 2014 